\title{
DENDROIDAL SETS AS MODELS FOR HOMOTOPY OPERADS
}

\author{
DENIS-CHARLES CISINSKI AND IEKE MOERDIJK
}

\begin{abstract}
The homotopy theory of $\infty$-operads is defined by extending Joyal's homotopy theory of $\infty$-categories to the category of dendroidal sets. We prove that the category of dendroidal sets is endowed with a model category structure whose fibrant objects are the $\infty$-operads (i.e. dendroidal inner Kan complexes). This extends the theory of $\infty$-categories in the sense that the Joyal model category structure on simplicial sets whose fibrant objects are the $\infty$-categories is recovered from the model category structure on dendroidal sets by simply slicing over the point.
\end{abstract}

\section{Contents}

Introduction

1. Dendroidal sets

2. Statement of main results 6

3. Construction of an abstract model category for $\infty$-operads 9

4. The join operation on trees 13

5. Subdivision of cylinders 21

6. $\infty$-operads as fibrant objects 26

Appendices 34

A. Grafting orders onto trees 34

B. Another subdivision of cylinders 38

Erratum 43

References

\section{INTRODUCTION}

The notion of dendroidal set is an extension of that of simplicial set, suitable for defining and studying nerves of (coloured) operads in the same way as nerves of categories feature in the theory of simplicial sets. It was introduced by one of the authors and I. Weiss in [MW07. As explained in that paper, the category dSet of dendroidal sets carries a symmetric monoidal structure, which is closely related to the Boardman-Vogt tensor product for operads BV73. There is also a corresponding internal Hom of dendroidal sets. The category of dendroidal sets

2000 Mathematics Subject Classification. 55P48, 55U10, 55U40, 18D10, 18D50, 18 G30.

Key words and phrases. Inner Kan complex, operad, $\infty$-operad, dendroidal set, $\infty$-category, quasi-category, simplicial set. 
extends the category $s \mathcal{S e t}$ of simplicial sets, in the precise sense that there are adjoint functors (left adjoint on the left)

$$
i_{!}: s \text { Set } \rightleftarrows d S e t: i^{*}
$$

with good properties. In particular, the functor $i_{\text {! }}$ is strong monoidal and fully faithful, and identifies $s \mathcal{S} e t$ with the slice category $d \mathcal{S} e t / \eta$, where $\eta$ is the unit of the monoidal structure on $d S$ Set. (In fact, this adjunction is an open embedding of toposes.)

Using these adjoint functors $i_{\text {! }}$ and $i^{*}$, we can say more precisely how various constructions and results from the theory of simplicial sets extend to that of dendroidal sets. For example, the nerve functor $N:$ Cat $\longrightarrow s S e t$ and its left adjoint, which we denote by $\tau$, naturally extend to a pair of adjoint functors

$$
\tau_{d}: d \text { Set } \rightleftarrows \text { Operad }: N_{d}
$$

which plays a central role in our work.

The goal of this paper is to lay the foundations for a homotopy theory of dendroidal sets and " $\infty$-operads" (or "operads-up-to-homotopy", or "quasi-operads") which extends the simplicial theory of $\infty$-categories (or quasi-categories) which has recently been developed by Joyal, Lurie and others. Our main result is the existence of a Quillen closed model structure on the category of dendroidal sets, having the following properties:

1. This Quillen model structure on $d$ Set is symmetric monoida 1 (in the sense of [Hov99]) and left proper;

2. The fibrant objects of this model structure are precisely the $\infty$-operads.

3. The induced model structure on the slice category $d \mathcal{S}$ et $/ \eta$ is precisely the Joyal model structure on simplicial sets [JT07, Lur06].

The existence of such a model structure was suggested in [MW07. The $\infty$-operads refered to in 2 . are the dendroidal analogues of the $\infty$-categories forming the fibrant objects in the Joyal model structure. They are the dendroidal sets satisfying a lifting condition analogous to the weak Kan condition of Boardman-Vogt, and were introduced in [MW07, MW09] under the name "(dendroidal) inner Kan complexes". The dendroidal nerve of every operad is such an $\infty$-operad; conversely, intuitively speaking, $\infty$-operads are operads in which the composition of operations is only defined up to homotopy, in a way which is associative up to homotopy. For example, the homotopy coherent nerve of a symmetric monoidal topological category is an $\infty$-operad. The theory of $\infty$-operads contains the theory of $\infty$-categories, as well as the theory of symmetric monoidal $\infty$-categories and of operads in them. The theory of $\infty$-operads is also likely to be of use in studying the notion of $\infty$-category enriched in a symmetric monoidal $\infty$-category (e.g. the various notions of $A_{\infty^{-}}$ categories, dg categories, weak $n$-categories).

The proof of our main theorem is based on three sources: First of all, we use the general methods of constructing model structures on presheaf categories developed in [Cis06] (we only use the first chapter and Section 8.1 of that book, which are both elementary). Secondly, we use some fundamental properties of dendroidal inner Kan complexes proved in MW09. And finally, we use some important notions and

\footnotetext{
${ }^{1}$ This model category structure is not monoidal; see the Erratum at the end of these notes, where we explain that this does not affect the main results of this paper nor of its sequels. We have chosen not to modify the present article to keep it as close as possible to the published version.
} 
results from Joyal's seminal paper [Joy02: namely, the theory of join operations and the notions of left or right fibration of simplicial sets. Apart from these sources, our proof is entirely self-contained. In particular, we do not use the Joyal model structure in our proof, but instead deduce this model structure as a corollary, as expressed in 3. above.

It is known that there are several (Quillen) equivalent models for $\infty$-categories: one is given by a left Bousfield localisation of the Reedy model structure on simplicial spaces and has as its fibrant objects Rezk's complete Segal spaces; another is given by a Dwyer-Kan style model structure on topological categories established by Bergner, in which all objects are fibrant. The equivalence of these approaches is extensively discussed in Lurie's book Lur06; see also [Ber07, JT07. It is natural to ask whether analogous models exist for $\infty$-operads. In two subsequent papers [CM13a, CM13b], we will show that this is indeed the case. We will prove there that the model structure on dendroidal sets described above is equivalent to a model structure on topological operads in which all objects are fibrant, as well as to a model structure on dendroidal spaces whose fibrant objects are "dendroidal complete Segal spaces". The models for $\infty$-categories just mentioned as well as the equivalences between them will again emerge simply by slicing over suitable unit objects of the respective monoidal structures. Together these model categories fit into a row of Quillen equivalences

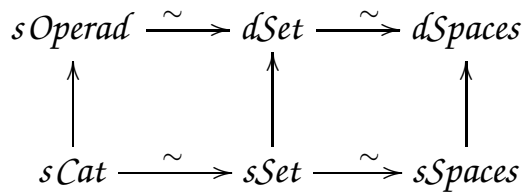

in which the vertical arrows are (homotopy) full embeddings.

This paper is organized as follows. In the first section, we recall the basics about dendroidal sets. In Section 2, we state the main results of this paper: the existence of a model category structure on the category of dendroidal sets whose fibrant objects are the $\infty$-operads, as well as its main properties. In Section 3, we construct this model structure through rather formal arguments. At this stage, it is clear, by construction, that the fibrant objects are $\infty$-operads, but the converse is not obvious. Sections 4 and 5 provide the tools to prove that any $\infty$-operad is fibrant, following the arguments which are known to hold in the case of simplicial sets for the theory of $\infty$-categories. More precisely, in Section 4, we develop a dendroidal analog of Joyal's join operations, and prove a generalization of a theorem of Joyal which ensures a right lifting property for inner Kan fibrations with respect to certain non-inner horns, under an additional hypothesis of weak invertibility of some 1-cells. In Section 5, we construct and examine a subdivision of cylinders of trees in terms of dendroidal horns. At last, in Section 6, we prove that any $\infty$-operad is fibrant, and study some of the good properties of fibrations between $\infty$-operads. This is done by proving an intermediate result which is important by itself: a morphism of diagrams in an $\infty$-operad is weakly invertible if and only if it is locally (i.e. objectwise) weakly invertible (this is where Sections 4 and 5 have their roles to play).

We also added two appendices, which are independent of the rest of this paper. In Appendix A we study the join operations on leaves (while in Section 4, we studied join operations on roots), and in Appendix B, we study another subdivision of 
cylinders of trees. In fact, these appendices can be used to provide another proof of our main results: Section 6 might have been written using Appendices $\mathrm{A}$ and B instead of Sections 4 and 5 respectively, without any changes (except, sometimes, replacing the evaluation by 1 by the evaluation by 0 , whenever necessary). However, these appendices are not formal consequences of the rest ot these notes, and it will be useful to have this kind of results available for further work on the subject.

\section{Dendroidal Sets}

1.1. Recall from [MW07] the category of trees $\Omega$. The objects of $\Omega$ are non-empty non-planar trees with a designated root, and given two trees $T$ and $T^{\prime}$, a map from $T$ to $T^{\prime}$ is a morphism of the corresponding operads which, in these notes, we will denote by $T$ and $T^{\prime}$ again. Hence, by definition, the category of trees is a full subcategory of the category of operads. Recall that the category $d S e t$ of dendroidal sets is defined as the category of presheaves of sets on the category of trees $\Omega$. Given a tree $T$, we denote by $\Omega[T]$ the dendroidal set represented by $T$.

Let 0 be the tree with only one edge, and set $\eta=\Omega[0]$. Then the category $\Omega / \eta$ identifies canonically with the category $\Delta$ of simplices, so that the category $d \mathcal{S} e t / \eta$ is canonically equivalent to the category $s \mathcal{S} e t$ of simplicial sets. The corresponding functor

$$
i: \Delta \longrightarrow \Omega, \quad[n] \longmapsto i[n]=n
$$

is fully faithful and its image is a sieve in $\Omega$. This functor $i$ induces an adjunction

$$
i_{!}: s \mathcal{S} e t \rightleftarrows d \mathcal{S} e t: i^{*}
$$

(where $i_{\text {! }}$ is the left Kan extension of $i$ ). Under the identification $s \mathcal{S} e t=d \mathcal{S} e t / \eta$, the functor $i_{\text {! }}$ is simply the forgetful functor from $d S e t / \eta$ to $d S e t$. The functor $i_{\text {! }}$ is fully faithful and makes sSet into an open subtopos of $d S e t$. In other words, if there is a map of dendroidal sets $X \longrightarrow Y$ with $Y$ a simplicial set, then $X$ has to be a simplicial set as well.

We also recall the pairs of adjoint functors

$$
\tau: \text { sSet } \rightleftarrows \text { Cat }: N \quad \text { and } \quad \tau_{d}: \text { dSet } \rightleftarrows \text { Operad }: N_{d}
$$

where $N$ and $N_{d}$ denote the nerve functors from the category of categories to the category of simplicial sets and from the category of (symmetric coloured) operads to the category of dendroidal sets.

The category of operads is endowed with a closed symmetric monoidal structure: the tensor product is defined as the Boardman-Vogt tensor product; see [MW07. Section 5]. This defines canonically a unique closed symmetric monoidal structure on the category of dendroidal sets such that the functor $\tau_{d}$ is symmetric monoidal, and such that, for two trees $T$ and $S$, we have

$$
\Omega[T] \otimes \Omega[S]=N_{d}\left(T \otimes_{B V} S\right),
$$

where $T \otimes_{B V} S$ is the Boardman-Vogt tensor product of operads. We will denote internal Hom objects by $\mathcal{H o m}(A, X)$ or by $X^{A}$.

Note that the functor $i_{!}: s \mathcal{S} e t \longrightarrow d \mathcal{S e t}$ is a symmetric monoidal functor, if we consider $s \mathcal{S} e t$ with its closed cartesian monoidal structure.

The functor $i^{*}$ turns the category of dendroidal sets into a simplicial category; given two dendroidal sets $A$ and $X$, we will write $\operatorname{fom}(A, X)$ for $i^{*}(\mathcal{H o m}(A, X))$, the simplicial set of maps from $A$ to $X$. 
1.2. We recall here from [MW07] the different kinds of faces of trees in $\Omega$.

Let $T$ be a tree.

If $e$ is an inner edge of $T$, we will denote by $T / e$ the tree obtained from $T$ by contracting $e$. We then have a canonical inclusion

$$
\partial_{e}: T / e \longrightarrow T \text {. }
$$

A map of type (1.2.1) is called an inner face of $T$.

If $v$ is a vertex of $T$, with the property that all but one of the edges incident to $v$ are outer, we will denote by $T / v$ the tree obtained from $T$ by removing the vertex $v$ and all the outer edges incident to it. We then have a canonical inclusion

$$
\partial_{v}: T / v \longrightarrow T \text {. }
$$

A map of type (1.2.2) is called an outer face of $T$.

A map of type (1.2.1) or (1.2.2) will be called an elementary face of $T$.

We define $\partial \Omega[T]$ as the union in $d S e t$ of all the images of elementary face maps $\Omega[T / x] \longrightarrow \Omega[T]$. We thus have, by definition, an inclusion

$$
\partial \Omega[T] \longrightarrow \Omega[T] .
$$

Maps of shape (1.2.3) are called boundary inclusions. The image of a face map $\partial_{x}$ will sometimes be denoted by $\partial_{x}(T)$ for short.

We will call faces the maps of $\Omega$ which are obtained, up to an isomorphism, as compositions of elementary faces. It can be checked that faces are exactly the monomorphisms in $\Omega$; see [MW07, Lemma 3.1].

1.3. A monomorphism of dendroidal sets $X \longrightarrow Y$ is normal if for any tree $T$, any non degenerate dendrex $y \in Y(T)$ which does not belong to the image of $X(T)$ has a trivial stabilizer $\operatorname{Aut}(T)_{y} \subset \operatorname{Aut}(T)$. A dendroidal set $X$ is normal if the map $\emptyset \longrightarrow X$ is normal. For instance, for any tree $T$, the dendroidal set $\Omega[T]$ is normal.

Proposition 1.4. The class of normal monomorphisms is stable by pushouts, transfinite compositions and retracts. Furthermore, this is the smallest class of maps in dSet which is closed under pushouts and tranfinite compositions, and which contains the boundary inclusions $\partial \Omega[T] \longrightarrow \Omega[T], T \in \Omega$.

Proof. This follows from [Cis06, Proposition 8.1.35].

Proposition 1.5. A monomorphism of dendroidal sets $X \longrightarrow Y$ is normal if and only if for any tree $T$, the action of $\operatorname{Aut}(T)$ on $Y(T)-X(T)$ is free.

Proof. It is easily seen that the class of monomorphisms which satisfy the above property is stable by pushouts and transfinite compositions, and contains the boundary inclusions $\partial \Omega[T] \longrightarrow \Omega[T]$. It thus follows from the preceding proposition that any normal monomorphism has this property. But it is also obvious that any monomorphism with this property is normal.

Corollary 1.6. A dendroidal set $X$ is normal if and only if for any tree $T$, the action of the group $\operatorname{Aut}(T)$ on $X(T)$ is free.

Corollary 1.7. Given any map of dendroidal sets $X \longrightarrow Y$, if $Y$ is normal, then $X$ is normal.

Corollary 1.8. Any monomorphism $i: A \longrightarrow B$ with $B$ normal is a normal monomorphism. 
Proposition 1.9. Let $A \longrightarrow B$ and $X \longrightarrow Y$ be two normal monomorphisms. The induced map

$$
A \otimes Y \amalg_{A \otimes X} B \otimes X \longrightarrow B \otimes Y
$$

is a normal monomorphism.

Proof. As the class of normal monomorhisms is generated by the boundary inclusions, it is sufficient to check this property in this case; see e.g. Hov99, Lemma 4.2.4].

Consider now two trees $S$ and $T$. We have to show that the map

$$
\partial \Omega[S] \otimes \Omega[T] \amalg_{\partial \Omega[S] \otimes \partial \Omega[T]} \Omega[S] \otimes \partial \Omega[T] \longrightarrow \Omega[S] \otimes \Omega[T]
$$

is a normal monomorphism. But as $\Omega[S] \otimes \Omega[T]$ is the dendroidal nerve of the Boardman-Vogt tensor product of $S$ and $T$, which is $\Sigma$-free, it is a normal dendroidal set. Hence we are reduced to prove that the above map is a monomorphism. This latter property is equivalent to the fact that the commutative square

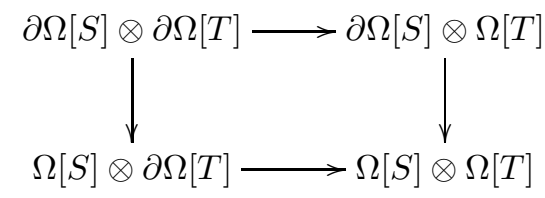

is a pullback square in which any map is a monomorphism. As the nerve functor preserves pullbacks, this reduces to the following property: for any elementary faces $S / x \longrightarrow S$ and $T / y \longrightarrow T$ the commutative square

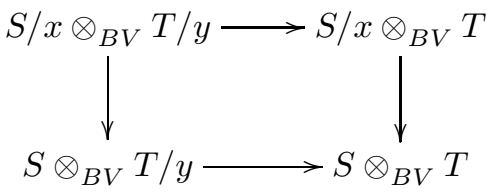

is a pullback square of monomorphisms in the category of operads. This is an elementary consequence of the definitions involved.

1.10. Under the assumtions of Proposition 1.9, we shall write $A \otimes Y \cup B \otimes X$ instead of $A \otimes Y \amalg_{A \otimes X} B \otimes X$.

\section{Statement of MAin RESUlts}

In this section, we state the main results of this paper.

2.1. Recall from [MW09, Section 5] the notion of inner horn. Given an inner edge $e$ in a tree $T$, we get an inclusion

$$
\Lambda^{e}[T] \longrightarrow \Omega[T],
$$

where $\Lambda^{e}[T]$ is obtained as the union of all the images of elementary face maps which are distinct from the face $\partial_{e}: T / e \longrightarrow T$. The maps of shape (2.1.1) are called inner horn inclusions.

A map of dendroidal sets is called an inner anodyne extension if it belongs to the smallest class of maps which is stable by pushouts, transfinite composition and retracts, and which contains the inner horn inclusions.

A map of dendroidal sets is called an inner Kan fibration if it has the right lifting property with respect to the class of inner anodyne extensions (or, equivalently, to the set of inner horn inclusions). 
A dendroidal set $X$ is an inner Kan complex if the map from $X$ to the terminal dendroidal set is an inner Kan fibration. We will also call inner Kan complexes $\infty$ operads. For example, for any operad $\mathcal{P}$, the dendroidal set $N_{d}(\mathcal{P})$ is an $\infty$-operad; see [MW09, Proposition 5.3]. In particular, for any tree $T$, the dendroidal set $\Omega[T]$ is an $\infty$-operad. For a simplicial set $K$, its image by $i_{!}$is an $\infty$-operad if and only if $K$ is an $\infty$-category (i.e. $K$ is a quasi-category in the sense of Joy02 ).

A map of dendroidal sets will be called a trivial fibration if it has the right lifting property with respect to normal monomorphisms.

Note that the small object argument implies that we can factor any map of dendroidal sets into a normal monomorphism followed by a trivial fibration (resp. into an inner anodyne extension followed by an inner Kan fibration).

Remark 2.2. A morphism between normal dendroidal sets is a trivial fibration if and only if it has the right lifting property with respect to monomorphisms: this follows immediately from Corollaries 1.7 and 1.8 .

2.3. Recall the naive model structure on the category of operads [Wei07]: the weak equivalences are the equivalences of operads, i.e. the maps $f: \mathcal{P} \longrightarrow Q$ which are fully faithful and essentially surjective: for any $n+1$-uple of objects $\left(a_{1}, \ldots, a_{n}, a\right)$ in $\mathcal{P}, f$ induces a bijection

$$
\mathcal{P}\left(a_{1}, \ldots, a_{n} ; a\right) \longrightarrow Q\left(f\left(a_{1}\right), \ldots, f\left(a_{n}\right) ; f(a)\right),
$$

and any object of $Q$ is isomorphic to the image of some object in $\mathcal{P}$. The fibrations are operadic fibrations, i.e. the maps $f: \mathcal{P} \longrightarrow Q$ such that, given any isomorphism $\beta: b_{0} \longrightarrow b_{1}$ in $Q$, and any object $a_{1}$ in $\mathcal{P}$ such that $f\left(a_{1}\right)=b_{1}$, there exists an isomorphism $\alpha: a_{0} \longrightarrow a_{1}$ in $\mathcal{P}$, such that $f(\alpha)=\beta$.

This model structure is closely related with the naive model structure on Cat (for which the weak equivalences are the equivalences of categories). In fact, the latter can be recovered from the one on operads by slicing over the unit operad (which is also the terminal category). The fibrations of the naive model structure on Cat will be called the categorical fibrations.

Theorem 2.4. The category of dendroidal sets is endowed with a model category structure for which the cofibrations are the normal monomorphisms, the fibrant objects are the $\infty$-operads, and the fibrations between fibrant objects are the inner Kan fibrations between $\infty$-operads whose image by $\tau_{d}$ is an operadic fibration. The class of weak equivalences is the smallest class of maps of dendroidal sets $\mathrm{W}$ which satisfies the following three properties.

(a) ('2 out 3 property') In any commutative triangle, if two maps are in W, then so is the third.

(b) Any inner anodyne extension is in $\mathrm{W}$.

(c) Any trivial fibration between $\infty$-operads is in $\mathrm{W}$.

Proof. This follows from Proposition 3.12 Theorem 6.10, and Corollary 6.11

Corollary 2.5. The adjunction $\tau_{d}:$ dSet $\rightleftarrows$ Operad : $N_{d}$ is a Quillen pair. Moreover, the two functors $\tau_{d}$ and $N_{d}$ both preserve weak equivalences. In particular, a morphism of operads is an equivalence of operads if and only if its dendroidal nerve is a weak equivalence.

Proof. See6.17. 
Proposition 2.6. The model category structure of Theorem 2.4 has the following additional properties:

(a) it is left proper;

(b) it is cofibrantly generated (it is even combinatorial);

(c) it is symmetric monoidal.

Proof. See Propositions 3.12 and 3.17

Corollary 2.7. For any normal dendroidal set $A$ and any $\infty$-operad $X$, the set of maps $[A, X]=\operatorname{Hom}_{\mathbf{H o}(d S e t)}(A, X)$ is canonically identified with the set of isomorphism classes of objects in the category $\tau$ from $(A, X)$.

Proof. See Proposition 6.20.

Corollary 2.8. Let $f: X \longrightarrow Y$ be a morphism of $\infty$-operads. The following conditions are equivalent.

(a) The map $f: X \longrightarrow Y$ is a weak equivalence.

(b) For any normal dendroidal set $A$, the map

$$
\tau_{d} \mathcal{H o m}(A, X) \longrightarrow \tau_{d} \mathcal{H o m}(A, Y)
$$

is an equivalence of operads.

(c) For any normal dendroidal set $A$, the map

$$
\tau \operatorname{fom}(A, X) \longrightarrow \tau \operatorname{hom}(A, Y)
$$

is an equivalence of categories.

Proof. Remember that, by definition (and any $\infty$-operad being fibrant), the map $f$ is a weak equivalence if and only if, for any normal dendroidal set $A$, the induced map

$$
[A, X] \longrightarrow[A, Y]
$$

is bijective. This corollary is thus a direct consequence of Corollaries 2.5 and 2.7 and of the fact the model category structure on $d \mathcal{S} e t$ is monoidal.

Corollary 2.9. Let $u: A \longrightarrow B$ be a morphism of normal dendroidal sets. The following conditions are equivalent.

(a) The map $u: A \longrightarrow B$ is a weak equivalence.

(b) For any $\infty$-operad $X$, the map

$$
\tau_{d} \operatorname{Hom}(B, X) \longrightarrow \tau_{d} \operatorname{Hom}(A, X)
$$

is an equivalence of operads.

(c) For any $\infty$-operad $X$, the map

$$
\tau \operatorname{from}(B, X) \longrightarrow \tau \operatorname{from}(A, X)
$$

is an equivalence of categories.

Proof. The fibrant objects of $d S e t$ are exactly the $\infty$-operads. Hence, the map $u: A \longrightarrow B$ is a weak equivalence if and only if, for any $\infty$-operad $X$, the map

$$
[B, X] \longrightarrow[A, X]
$$

is bijective. We conclude the proof using the same arguments as in the proof of Corollary 2.8. 
Corollary 2.10 (Joyal). The category of simplicial sets is endowed with a left proper, cofibrantly generated, symmetric monoidal model category structure for which the cofibrations are the monomorphisms, the fibrant objects are the $\infty$-categories, and the fibrations between fibrant objects are the inner Kan fibrations between $\infty$-categories whose image by $\tau$ is a categorical fibration.

Proof. The model category structure on $d S e t$ induces a model category structure on $d$ Set $/ \eta \simeq s$ Set; see also Remark 3.14 for $B=\eta$.

Remark 2.11. Note that, the functor $i_{!}: s$ Set $\longrightarrow d S e t$ is fully faithful and symmetric monoidal. Moreover, for any simplicial sets $A$ and $X$, we have fom $\left(i_{!}(A), i_{!}(X)\right)=$ $X^{A}$. We deduce from this that the induced map

$$
\operatorname{Hom}_{\mathbf{H o}(s S e t)}(A, X) \longrightarrow \operatorname{Hom}_{\mathbf{H o}(d S e t)}\left(i_{!}(A), i_{!}(X)\right)
$$

is bijective (where $\mathbf{H o}(s \mathcal{S e t}$ ) denotes the homotopy category of the Joyal model structure, given by Corollary 2.10). As a consequence, we also have formally the simplicial analogs of Corollaries 2.7, 2.8 and 2.9.

\section{Construction of An Abstract model CAtegory for $\infty$-Operads}

This section is devoted to the construction of a model category structure on $d S$ Set. The construction is relatively formal and uses very little of the theory of dendroidal sets. By definition, we will have that any fibrant object of this model category is an $\infty$-operad. The proof of the converse (any $\infty$-operad is fibrant) is the 'raison d'être' of the next sections.

Proposition 3.1. Let $A \longrightarrow B$ and $X \longrightarrow Y$ be an inner anodyne extension and a normal monomorphism respectively. The induced map

$$
A \otimes Y \cup B \otimes X \longrightarrow B \otimes Y
$$

is an inner anodyne extension.

Proof. Using [Cis06, Corollary 1.1.8], we see that it is sufficient to check this property when $A \longrightarrow B$ is an inner horn inclusion and when $X \longrightarrow Y$ is a boundary inclusion. This proposition thus follows from [MW09, Proposition 9.2].

3.2. We denote by $J$ the nerve of the contractible groupoid with two objects 0 and 1 (i.e. $J$ is the nerve of the fundamental groupoid of $\Delta[1]$ ). We will write $J_{d}=i_{!}(J)$ for the corresponding dendroidal set.

A morphism of dendroidal sets is a $J$-anodyne extension if it belongs to the smallest class of maps which contains the inner anodyne extensions and the maps

$$
\partial \Omega[T] \otimes J_{d} \cup \Omega[T] \otimes\{e\} \longrightarrow \Omega[T] \otimes J_{d} \quad T \in \Omega, e=0,1,
$$

and which is closed under pushouts, transfinite compositions and retracts.

A morphism of dendroidal sets will be called a $J$-fibration if it has the right lifting property with respect to $J$-anodyne extensions.

A dendroidal set $X$ is $J$-fibrant if the map from $X$ to the terminal dendroidal set is a $J$-fibration.

Proposition 3.3. Let $A \longrightarrow B$ and $X \longrightarrow Y$ be a J-anodyne extension and $a$ normal monomorphism respectively. The induced map

$$
A \otimes Y \cup B \otimes X \longrightarrow B \otimes Y
$$

is a $J$-anodyne extension. 
Proof. Using [Cis06, Corollary 1.1.8], this follows formally from the definition and from Proposition 3.1 .

3.4. Let $B$ be a dendroidal set. Denote by $A n_{B}$ the class of maps of $d S e t / B$ whose image in $d S e t$ is $J$-anodyne. For each dendroidal set $X$ over $B$, with structural map $a: X \longrightarrow B$, we define a cylinder of $X$ over $B$

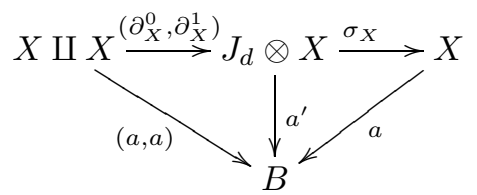

in which $\partial_{X}^{e}$ is the tensor product of $\{e\} \longrightarrow J_{d}$ with $1_{X}$, while $\sigma_{X}$ is the tensor product of $J_{d} \longrightarrow \eta$ with $1_{X}$, and $a^{\prime}$ is the composition of $1_{J_{d}} \otimes a$ with the map $\sigma_{B}$.

These cylinders over $B$ define the notion $J$-homotopy over $B$ (or fiberwise $J$ homotopy) between maps in $d \mathcal{S} e t / B$. Given two dendroidal sets $A$ and $X$ over $B$, we define $[A, X]_{B}$ as the quotient of the set $\operatorname{Hom}_{d \text { Set } / B}(A, X)$ by the equivalence relation generated by the relation of $J$-homotopy over $B$. A morphism $A \longrightarrow A^{\prime}$ of dendroidal sets over $B$ is a $B$-equivalence if, for any dendroidal set $X$ over $B$ such that the structural map $X \longrightarrow B$ is a $J$-fibration, the map

$$
\left[A^{\prime}, X\right]_{B} \longrightarrow[A, X]_{B}
$$

is bijective.

In the case $B$ is normal, any monomorphism over $B$ is normal; see Corollaries 1.7 and 1.8. We see from Proposition 3.3 and from Cis06, Lemma 1.3.52] that the class $\mathrm{An}_{B}$ is a class of anodyne extensions with respect to the functorial cylinder (3.4.1) in the sense of [Cis06, Definition 1.3.10]. In other words, the functorial cylinder (3.4.1) and the class $A_{B}$ form a homotopical structure on the category dSet / B in the sense of [Cis06, Definition 1.3.14]. As a consequence, a direct application of Cis06. Theorem 1.3.22, Proposition 1.3.36 and Lemma 1.3.52] leads to the following statement?

Proposition 3.5. For any normal dendroidal set B, the category dSet/B of dendroidal sets over $B$ is endowed with a left proper cofibrantly generated model category structure for which the weak equivalences are the B-equivalences, the cofibrations are the monomorphisms, and the fibrant objects are the dendroidal sets $X$ over $B$ such that the structural map is a J-fibration. Moreover, a morphism between fibrant objects is a fibration in $\mathbf{d S e t} / B$ if and only if its image in dSet is a J-fibration.

Remark 3.6. Any $J$-anodyne extension over $B$ is a trivial cofibration in the model structure of the preceding proposition; see [Cis06, Proposition 1.3.31].

Lemma 3.7. Let $p: X \longrightarrow Y$ be a trivial fibration between normal dendroidal sets. Any section $s: Y \longrightarrow X$ is a $J$-anodyne extension.

Proof. This is a particular case of [Cis06, Corollary 1.3.35] applied to the homotopical structure defined in 3.4 on $d \mathcal{S e t} / Y$.

\footnotetext{
${ }^{2}$ The results of [Cis06] are stated for presheaves categories, so that, strictly speaking, to apply them, we implicitely use the canonical equivalence of categories between $d \mathcal{S}$ et $/ B$ and the category of presheaves on $\Omega / B$.
} 
3.8. We fix once and for all a normalization $E_{\infty}$ of the terminal dendroidal set: i.e., we choose a normal dendroidal set $E_{\infty}$ such that the map from $E_{\infty}$ to the terminal dendroidal set is a trivial fibration.

Lemma 3.9. For any normal dendroidal set $X$, and any map $a: X \longrightarrow E_{\infty}$, the map $\left(a, 1_{X}\right): X \longrightarrow E_{\infty} \times X$ is a J-anodyne extension.

Proof. This follows immediately from Lemma 3.7 because $\left(a, 1_{X}\right)$ is a section of the projection $X \times E_{\infty} \longrightarrow X$, which is a trivial fibration by definition of $E_{\infty}$.

Lemma 3.10. Let $i: A \longrightarrow B$ be a morphism of normal dendroidal sets, and $p: X \longrightarrow Y$ a morphism of dendroidal sets. The map $p$ has the right lifting property with respect to $i$ in dSet if and only if, for any morphism $B \longrightarrow E_{\infty}$, the map $1_{E_{\infty}} \times p$ has the right lifting property with respect to $i$ in $d S e t / E_{\infty}$.

Proof. Suppose that $1_{E_{\infty}} \times p$ has the right lifting property with respect to $i$, and consider the lifting problem below.

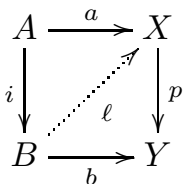

As $B$ is normal, there exists a map $\beta: B \longrightarrow E_{\infty}$. If we write $\alpha=\beta i$, we see immediately that the lifting problem above is now equivalent to the lifting problem

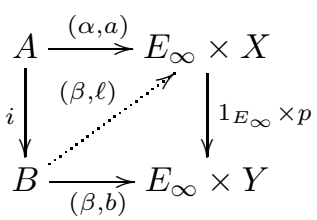

and this proves the lemma.

3.11. Given a normal dendroidal set $A$ and a $J$-fibrant dendroidal set $X$, we denote by $[A, X]$ the quotient of $\operatorname{Hom}_{d \mathcal{S} e t}(A, X)$ by the equivalence relation generated by the $J$-homotopy relation (i.e., with the notations of [3.4. $[A, X]=[A, X]_{e}$, where $e$ denotes the terminal dendroidal set).

Proposition 3.12. The category of dendroidal sets is endowed with a left proper cofibrantly generated model category in which the cofibrations are the normal monomorphisms, the fibrant objects are the $J$-fibrant dendroidal sets, and the fibrations between fibrant objects are the $J$-fibrations. Furthermore, given a normal dendroidal set $A$ and a $J$-fibrant dendroidal set $X$, we have a canonical identification

$$
[A, X]=\operatorname{Hom}_{\mathbf{H o}(d \mathcal{S} e t)}(A, X) .
$$

Proof. Proposition 3.5 applied to $B=E_{\infty}$ gives us a model category structure on $d$ Set $/ E_{\infty}$. Consider the adjunction

$$
p_{!}: d \operatorname{Set} / E_{\infty} \rightleftarrows d S e t: p^{*},
$$

where $p^{*}$ is the functor $X \longmapsto E_{\infty} \times X$. It follows obviously from Lemma 3.9 that the functor $p^{*} p_{\text {! }}$ is a left Quillen equivalence from the category $d \mathcal{S} e t / E_{\infty}$ to itself. This implies immediately that the adjunction $\left(p_{!}, p^{*}\right)$ satisfies all the necessary hypothesises to define a model structure on $d S$ et by transfer; see e.g. Cra95] 
or [Cis06, Proposition 1.4.23]. In other words, the category of dendroidal sets is endowed with a cofibrantly generated model category structure for which the weak equivalences (resp. the fibrations) are the maps whose image by $p^{*}$ is a weak equivalence (resp. a fibration) in $d \mathcal{S} e t / E_{\infty}$. The description of cofibrations follows from Proposition 1.4. We know that the fibrations between fibrant objects in $d S e t / E_{\infty}$ are the maps whose image in $d S e t$ is a $J$-fibration; see Proposition 3.5. The description of fibrant objects and of fibrations between fibrant objects in $d$ Set as $J$-fibrant objects and $J$-fibrations is thus a direct consequence of Lemma 3.10. The identification $[A, X]=\operatorname{Hom}_{\mathbf{H o}(d S e t)}(A, X)$ is obtained from the general description of the set of maps from a cofibrant object to a fibrant object in an abstract model category. It remains to prove left properness: this follows from the left properness of the model category structure of Proposition 3.5 for $B=E_{\infty}$ (which is obvious, as any object over $E_{\infty}$ is cofibrant), and from the fact that $p^{*}$ preserves cofibrations as well as colimits, while it preserves and detects weak equivalences.

3.13. The weak equivalences of the model structure defined in Proposition 3.12 will be called the weak operadic equivalences.

Given a dendroidal set $A$, a normalization of $A$ is a trivial fibration $A^{\prime} \longrightarrow A$ with $A^{\prime}$ normal. For instance, the projection $E_{\infty} \times A \longrightarrow A$ is a normalization of $A$ (as $E_{\infty}$ is normal, it follows from Corollary 1.7 that $E_{\infty} \times A$ is normal). For a morphism of dendroidal sets $f: A \longrightarrow B$, the following conditions are equivalent.

(a) The map $f$ is a weak operadic equivalence.

(b) For any commutative square

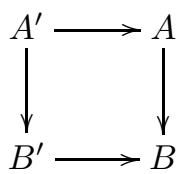

in which the horizontal maps are normalizations, and for any $J$-fibrant dendroidal set $X$, the map $\left[B^{\prime}, X\right] \longrightarrow\left[A^{\prime}, X\right]$ is bijective.

(c) There exists a commutative square

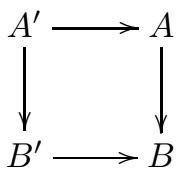

in which the horizontal maps are normalizations such that, for any $J$-fibrant dendroidal set $X$, the map $\left[B^{\prime}, X\right] \longrightarrow\left[A^{\prime}, X\right]$ is bijective.

Remark 3.14. Given a normal $J$-fibrant dendroidal set $B$, the model structure induced on $d \mathcal{S e t} / B$ by the model structure of Proposition 3.12 coincide with the model structure of Proposition [3.5 (this follows, for instance, from the fact these model structures have the same cofibrations and fibrations between fibrant objects).

Remark 3.15. The model category structure of Proposition 3.12 is cofibrantly generated. The generating cofibrations are the inclusions of shape $\partial \Omega[T] \longrightarrow \Omega[T]$ for any tree $T$. We don't know any explicit set of generating trivial cofibrations. However, we know (from the proof of Proposition 3.12) that there exists a generating set of trivial cofibrations $\mathcal{I}$ for the model structure on $d S e t / E_{\infty}$, such that 
$p_{!}(\mathcal{J})$ is a generating set of trivial cofibrations of $d \mathcal{S} e t$. In particular, there exists a generating set of trivial cofibrations of $d S e t$ which consists of trivial cofibrations between normal dendroidal sets. Statements about trivial cofibrations will often be reduced to statements about $J$-anodyne extensions using the following argument.

Proposition 3.16. The class of trivial cofibrations between normal dendroidal sets is the smallest class $\mathcal{C}$ of monomorphisms between normal dendroidal sets which contains J-anodyne extensions, and such that, given any monomorphisms between normal dendroidal sets

$$
A \stackrel{i}{\longrightarrow} B \stackrel{j}{\longrightarrow} C
$$

if $j$ and $j i$ are in $\mathcal{C}$, so is $i$.

Proof. Let $i: A \longrightarrow B$ be a monomorphism between normal dendroidal sets. As $B$ is normal, we can choose a map from $B$ to $E_{\infty}$. We can then choose a commutative diagram over $E_{\infty}$

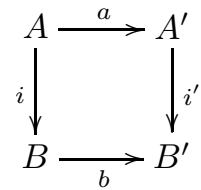

in which $a$ and $b$ are $J$-anodyne extensions, $A^{\prime}$ and $B^{\prime}$ are fibrant in $d S e t / E_{\infty}$, and $i^{\prime}$ is a monomorphism: this follows, for instance, from the fact that any $J$ fibrant resolution functor constructed with the small object argument applied to the generating set of $J$-anodyne extensions preserves monomorphisms; see Cis06, Proposition 1.2.35]. Applying Cis06, Corollary 1.3.35] to the model structure of Proposition 3.5 for $B=E_{\infty}$, we see that $i$ is a trivial cofibration if and only if $i^{\prime}$ is a $J$-anodyne extension. This proves the proposition.

Proposition 3.17. The model category structure on dSet is symmetric monoidal.

Proof. As we already know that normal monomorphisms are well behaved with respect to the tensor product (Proposition 1.9) it just remains to prove that, given a normal monomorphism $i: A \longrightarrow B$ and a trivial cofibration $j: C \longrightarrow D$, the induced map

$$
A \otimes D \cup B \otimes C \longrightarrow B \otimes D
$$

is a trivial cofibration. According to [Hov99, Lemma 4.2.4], we can assume that $i$ is a generating cofibration, and $j$ a generating trivial cofibration. In particular, we can assume that $i$ and $j$ are monomorphisms between normal dendroidal sets; see Remark 3.14. It is thus sufficient to prove that, given a normal dendroidal set $A$, the functor $X \longmapsto A \otimes X$ preserves trivial cofibrations between normal dendroidal sets. By Proposition 3.16, it is even sufficient to prove that tensor product by $A$ preserves $J$-anodyne extensions, which follows from Proposition 3.3 .

\section{The Join OPERATION ON TREeS}

The aim of this section is to study a dendroidal analog of the join operations on simplicial sets introduced by Joyal in Joy02. We shall prove a generalization of Joy02, Theorem 2.2]; see Theorem 4.2. 
4.1. Let $X$ be a $\infty$-operad. A 1 -simplex of $X$ (i.e. a map $\left.\Delta[1] \longrightarrow i^{*}(X)\right)$ will be called weakly invertible if the corresponding morphism in the category $\tau\left(i^{*}(X)\right)$ is an isomorphism.

Note that, for any $\infty$-operad $X$, the category $\tau\left(i^{*}(X)\right)$ is canonically isomorphic to the category underlying the operad $\tau_{d}(X)$ : this comes from the explicit description of $\tau\left(i^{*}(X)\right.$ given by Boardman and Vogt (see Joy02, Proposition 1.2]) and from its dendroidal generalization, which describes $\tau_{d}(X)$ explicitely; see [MW09, Proposition 6.10]. As a consequence, weakly invertible 1-cells in $X$ can be described as the maps $i_{!} \Delta[1]=\Omega[1] \longrightarrow X$ which induce invertible morphisms in the underlying category of the operad $\tau_{d}(X)$.

Theorem 4.2. Let $T$ be a tree with at least two vertices as well as a unary vertex $r$ at the root, and let $p: X \longrightarrow Y$ be an inner Kan fibration between $\infty$-operads. Then any solid commutative square of the form

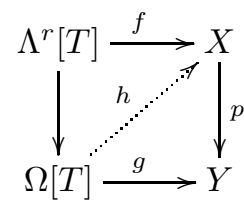

in which $f(r)$ is weakly invertible in $X$ has a diagonal filling $h$.

4.3. In order to prove this theorem, we will introduce join operations on forests.

A forest is a finite set of trees (i.e. of objects of $\Omega$ ). Given a forest $\mathcal{T}=$ $\left(T_{1}, \ldots, T_{k}\right), k \geqslant 0$, we write $\mathcal{T} / \mathcal{d} S$ et for the category of dendroidal sets under the coproduct $\Omega[\mathcal{T}]=\amalg_{i=1}^{k} \Omega\left[T_{i}\right]$. The objects of $\mathcal{T} /$ SSet are thus of shape $\left(X, x_{i}\right)=$ $\left(X, x_{1}, \ldots, x_{k}\right)$, where $X$ is a dendroidal set, and $x_{i} \in X\left(T_{i}\right)$, for $1 \leqslant i \leqslant k$. Morphisms $\left(X, x_{i}\right) \longrightarrow\left(Y, y_{i}\right)$ are maps $f: X \longrightarrow Y$ such that $f\left(x_{i}\right)=y_{i}$ for all $i$, $1 \leqslant i \leqslant k$.

Given an integer $n \geqslant 0$, we construct the tree $\mathcal{T} \star n$ by joining the trees $T_{1}, \cdots, T_{k}$ together over a new vertex $v$, and then grafting the result onto $i[n]$ (i.e. onto $[n]$ viewed as a tree).

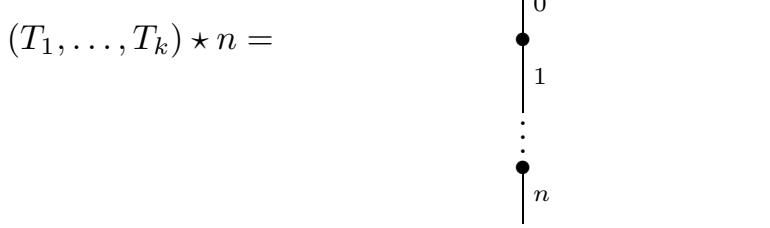


We insist that the forest $\mathcal{T}$ might be empty: for $k=0$, we have

$$
() \star n=\quad \begin{aligned}
& { }^{v}{ }_{0} \\
& 1 \\
& \vdots \\
& i_{n}
\end{aligned}
$$

As each $T_{i}, 1 \leqslant i \leqslant k$, embeds canonically into $\mathcal{T} \star n$, we can view $\Omega[\mathcal{T} \star n]$ as an object of $\mathcal{T} / \mathcal{d S e t}$. One checks that there is a unique functor

$$
\Delta \longrightarrow \Omega, \quad[n] \longmapsto \mathcal{T} \star n
$$

such that the inclusions $T_{i} \longrightarrow \mathcal{T} \star n$ are functorial in $T_{i}$ and such that the canonical inclusion $i[n] \longrightarrow \mathcal{T} \star n$ is functorial in $[n]$. This defines a functor

$$
\mathcal{T} \star(-): \Delta \longrightarrow \mathcal{T} / d \text { Set } .
$$

By Kan extension, we obtain a colimit preserving functor which extends (4.3.3):

$$
\mathcal{T} \star(-): s \text { Set } \longrightarrow \mathcal{T} / \text { dSet } .
$$

We have $\mathcal{T} \star \Delta[n]=\Omega[\mathcal{T} \star n]$. The functor (4.3.4) has a right adjoint

$$
\mathcal{T} \backslash(-): \mathcal{T} / d \text { Set } \longrightarrow \text { sSet } .
$$

For a one tree forest $\mathcal{T}=(T)$, we will simply write $\mathcal{T} \star K=T \star K$ and $\mathcal{T} \backslash X=$ $T \backslash X$ for any simplicial set $K$ and any dendroidal set $X$ under $\Omega[T]$. Under these conventions, these operations extend the join operations introduced by Joyal in Joy02 in the sense that we have the following formulas.

$$
\begin{aligned}
i[n] \star i_{!}(K) & =i_{!}(\Delta[n] \star K) \\
i[n] \backslash i_{!}(L) & =i_{!}(\Delta[n] \backslash L)
\end{aligned}
$$

Note that the inclusions $\Omega[n] \longrightarrow \mathcal{T} \star \Delta[n]$ in $d$ Set induce a natural projection map

$$
\pi_{X}: \mathcal{T} \backslash X \longrightarrow i^{*}(X)
$$

for any dendroidal set $X$ under $\mathcal{T}$.

Remark 4.4. Note that any tree with at least one vertex $T$ is obtained by joining a forest with an ordinal, i.e. as $T=\mathcal{T} \star n$ for some forest $\mathcal{T}$ and some integer $n \geqslant 0$. A tree $T$ has at least two vertices and a unary vertex at the root (as in the statement of Theorem 4.2) if and only if there exists a forest $\mathcal{T}$ such that $T=\mathcal{T} \star 1$.

4.5. In order to prove Theorem 4.2, we will have also to consider some specific maps of forests. For this purpose, we introduce the following terminology.

Let $T$ be a tree. A set $A$ of edges in $T$ is is called admissible if, for any input edge $e$ of $T$, and any vertex $v$ in $T$, if $A$ contains a path (branch) from $e$ to $v$, then $A$ contains all the edges above $v$.

If $A$ is an admissible set of edges in $T$, we will define a forest $\partial_{A}(T)$, and for each tree $S$ in $\partial_{A}(T)$, a face map $S \longrightarrow T$ in the category $\Omega$. Roughly speaking, one deletes from $T$ all edges in $A$, and defines $\partial_{A}(T)$ as the resulting connected components. A formal definition is by induction on the cardinality of $A$.

(i) If $A$ is empty, then $\partial_{A}(T)=T$. 
(ii) If $A$ contains the root edge $e$ of $T$, let $T_{1}, \ldots, T_{k}$ be the trees obtained from $T$ by deleting $e$ and the vertex immediately above it, let $A_{i}=T_{i} \cap A$, and define $\partial_{A}(T)$ as the union of the forests $\partial_{A_{i}}\left(T_{i}\right), 1 \leqslant i \leqslant k$.

(iii) If $A$ contains an input edge $a$ of $T$, it must contain all the edges above the vertex $v$ just below $a$. Let $T_{(v)}$ be the tree obtained from $T$ by pruning away $v$ and all the edges above it. Let $A_{(v)}=T_{(v)} \cap A$, and define $\partial_{A}(T)=$ $\partial_{A_{(v)}}\left(T_{(v)}\right)$.

(iv) If $A$ contains an inner edge $a$ of $T$, let $T / a$ be the tree obtained by contracting $a$, and define $\partial_{A}(T)$ to be $\partial_{A-\{a\}}(T / a)$.

One can check that the steps (i)-(iv) can be performed in any order, so that the forest $\partial_{A}(T)$ is well defined. Each tree $S$ in this forest $\partial_{A}(T)$ is a face of $T$, hence comes with a canonical map $S \longrightarrow T$.

Example 4.6. The tree

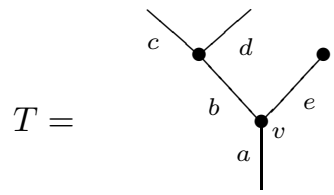

has two input edges $c$ and $d$. The edges $b$ and $c$ form a path from $c$ down to $v$. So any admissible set $A$ which contains $b$ and $c$, for example, must contain $d$ and $e$ as well.

4.7. This construction extends to forests in the following way. Let $\mathcal{T}=\left(T_{1}, \ldots, T_{k}\right)$ be a forest. An admissible subset of edges $A$ in $\mathcal{T}$ is a $k$-tuple $A=\left(A_{1}, \ldots, A_{k}\right)$, where $A_{i}$ is an admissible set of edges of $T_{i}$ for $1 \leqslant i \leqslant k$. We can then define the forest $\partial_{A}(T)$ as the union of the forests $\partial_{A_{i}}\left(T_{i}\right)$. Given any integer $n \geqslant 0$, we have a canonical map

$$
\partial_{A}(\mathcal{T}) \star n \longrightarrow \mathcal{T} \star n
$$

which is characterized by the fact that, given any tree $S$ in some $\partial_{A_{i}}\left(T_{i}\right)$, for $1 \leqslant i \leqslant k$, the diagram

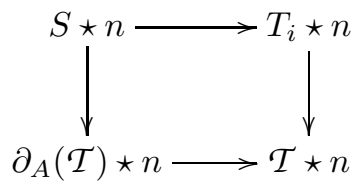

commutes. The map (4.7.1) is a monomorphism of trees in $\Omega$ and is natural in $[n]$ (as an object of $\Delta$ ). More generally, given an inclusion $A \subset B$ between admissible subsets of edges in $\mathcal{T}$, we have canonical monomorphisms of trees

$$
\partial_{B}(\mathcal{T}) \star n \longrightarrow \partial_{A}(\mathcal{T}) \star n
$$

(which is just another instance of (4.7.1) for the forest $\partial_{A}(\mathcal{T})$ with admissible subset of edges given by the sets $B_{i} \cap \partial_{A_{i}}\left(T_{i}\right)$ ). The maps (4.7.3) define a contravariant functor from the set of admissible subsets of edges in $\mathcal{T}$ (partially ordered by inclusion) to $\Omega$. Given an inclusion $A \subset B$ of admissible subsets of edges in $\mathcal{T}$, there exists a unique morphism

$$
\Omega\left[\partial_{B}(\mathcal{T})\right] \longrightarrow \Omega\left[\partial_{A}(\mathcal{T})\right]
$$


such that the following diagram commutes for any simplicial set $K$.

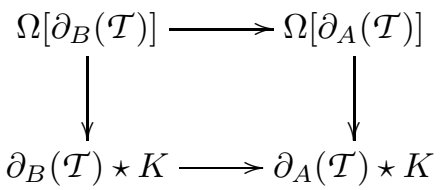

By adjunction, we also have natural morphisms

$$
\partial_{B}(\mathcal{T}) \backslash X \longrightarrow \partial_{A}(\mathcal{T}) \backslash X
$$

for all dendroidal sets $X$ under $\Omega\left[\partial_{A}(\mathcal{T})\right]$.

Example 4.8. If $a$ is the root of $T$, and if $T$ is obtained by grafting trees $T_{i}$ with root edges $a_{i}$ onto a corolla, then the map of type (4.7.3) for $A=\varnothing$ and $B=\{a\}$ is the map $\partial_{a}$ given by contracting $a$ :
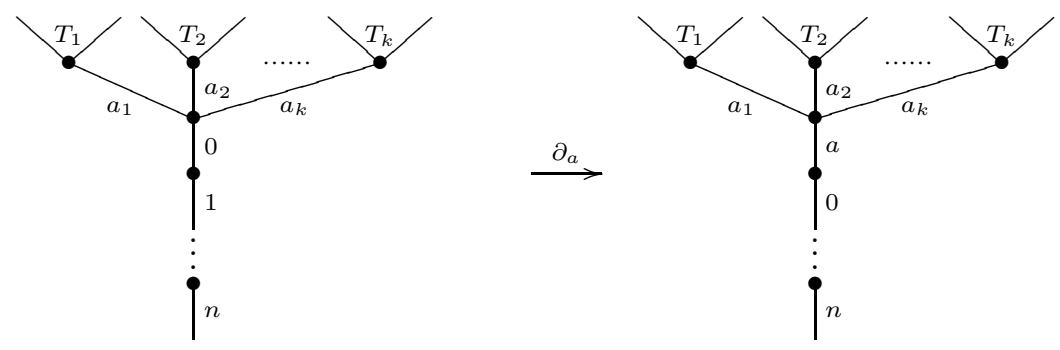

4.9. We will now study an elementary combinatorial situation which we will have to consider twice to prove Theorem 4.2 in the proof of Proposition 4.11] and in the proof of 4.15 .2 .

Consider a tree $T$. Assume that $T=\mathcal{T} \star n$ for a non-empty forest $\mathcal{T}=$ $\left(T_{1}, \ldots, T_{k}\right)$ and an ordinal $[n], n>0$.

Let $i, 0 \leqslant i<n$, be an integer, and $\left\{A_{1}, \ldots, A_{s}\right\}, s \geqslant 1$, a finite family of admissible subsets of edges in $\mathcal{T}$. Define

$$
C \subset D \subset \Omega[T]
$$

by

$$
C=\left(\bigcup_{r=1}^{s} \partial_{A_{r}}(\mathcal{T}) \star \Lambda^{i}[n]\right) \cup \Omega[n] \quad \text { and } \quad D=\bigcup_{r=1}^{s} \partial_{A_{r}}(\mathcal{T}) \star \Delta[n],
$$

where $\Omega[n]$ is considered as a subcomplex of $\Omega[T]$ through the canonical map.

Lemma 4.10. Under the assumptions of 4.9 , the map $C \longrightarrow D$ is an inner anodyne extension.

Proof. If $\mathcal{T}$ is the empty forest, we must have $s=1$ and $A_{1}=\varnothing$, so that $D=\Omega[T]$, and $C=\Lambda^{i}[T]$ is an inner horn. From now on, we will assume that $\mathcal{T}$ is non-empty.

Given a forest $\mathcal{T}^{\prime}$, the number of edges in $\mathcal{T}^{\prime}$ is simply defined as the sum of the number of edges in each of the trees which occur in $\mathcal{T}^{\prime}$. For each integer $p \geqslant 0$, write $\mathcal{F}_{p}$ for the set of faces $F$ which belong to $D$ but not to $C$, and which are of shape $F=\Omega\left[\partial_{A}(\mathcal{T}) \star n\right]$ for an admissible subset of edges $A$ in $\mathcal{T}$, such that $\partial_{A}(\mathcal{T})$ has exactly $p$ edges.

Define a filtration

$$
C=C_{0} \subset C_{1} \subset \ldots \subset C_{p} \subset \ldots \subset D
$$


by

$$
C_{p}=C_{p-1} \cup \bigcup_{F \in \mathcal{F}_{p}} F, \quad p \geqslant 1 .
$$

We have $D=C_{p}$ for $p$ big enough, and it is sufficient to prove that the inclusions $C_{p-1} \longrightarrow C_{p}$ are inner anodyne for $p \geqslant 1$. If $F$ and $F^{\prime}$ are in $\mathcal{F}_{p}$, then $F \cap F^{\prime}$ is in $C_{p-1}$. Moreover, if $F=\Omega\left[\partial_{A}(\mathcal{T}) \star n\right]$ for an admissible subset of edges $A$, then we have

$$
F \cap C_{p-1}=\Lambda^{i}\left[\partial_{A}(\mathcal{T}) \star n\right],
$$

which is an inner horn. Hence we can describe the inclusion $C_{p-1} \longrightarrow C_{p}$ as a finite composition of pushouts by inner horn inclusions of shape $F \cap C_{p-1} \longrightarrow F$ for $F \in \mathcal{F}_{p}$.

Proposition 4.11. Let $\mathcal{T}=\left(T_{1}, \ldots, T_{k}\right)$ be a forest, and $n \geqslant 1,0 \leqslant i<n$, be integers. The inclusion $\left(\mathcal{T} \star \Lambda^{i}[n]\right) \cup \Omega[n] \longrightarrow \mathcal{T} \star \Delta[n]$ is an inner anodyne extension.

Proof. This is a particular case of the preceding lemma.

4.12. Remember from Joy02 that a morphism of simplicial sets is called a left (resp. right) fibration if it has the right lifting property with respect to inclusions of shape $\Lambda^{i}[n] \longrightarrow \Delta[n]$ for $n \geqslant 1$ and $0 \leqslant i<n$ (resp. $0<i \leqslant n$ ).

A morphism between $\infty$-categories $X \longrightarrow Y$ is conservative if the induced functor $\tau(X) \longrightarrow \tau(Y)$ is conservative (which can be reformulated by saying that a 1-simplex of $X$ is weakly invertible if and only if its image in $Y$ is weakly invertible). For instance, by virtue of [Joy02, Proposition 2.7], any left (resp. right) fibration between $\infty$-categories is conservative.

Proposition 4.13. Let $p: X \longrightarrow Y$ be an inner Kan fibration of dendroidal sets under a forest $\mathcal{T}$. The map

$$
\mathcal{T} \backslash X \longrightarrow \mathcal{T} \backslash Y \times_{i^{*}(Y)} i^{*}(X)
$$

is a left fibration.

In particular, for any $\infty$-operad $X$ under a forest $\mathcal{T}$, the map $\mathcal{T} \backslash X \longrightarrow i^{*}(X)$ is a left fibration.

Proof. This follows immediately from Proposition 4.11 by a standard adjunction argument.

Corollary 4.14. For any $\infty$-operad $X$ and any forest $\mathcal{T}$ over $X$, the simplicial set $\mathcal{T} \backslash X$ is an $\infty$-category. Similarly, for any inner Kan fibration between $\infty$-operads $X \longrightarrow Y$ and any forest $\mathcal{T}$ over $X$, the simplicial set $\mathcal{T} \backslash Y \times{ }_{i^{*}(Y)} i^{*}(X)$ is an $\infty$-category.

Proof. If $X$ is an $\infty$-operad, then $i^{*}(X)$ is clearly an $\infty$-category. Since the projection $\mathcal{T} \backslash X \longrightarrow i^{*}(X)$ is a left fibration, this implies this corollary.

As a warm up to prove Theorem 4.2, we shall consider a particular case.

Lemma 4.15. Theorem 4.2 is true if $T=(\star \star 1$ (where ( ) denotes the empty forest). 
Proof. In this case, $T$ is a tree of shape

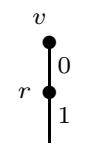

and $\Lambda^{r}[T]$ is the union of the two faces

$$
\dot{q}_{1} \text { and } r \rho_{1}^{0}
$$

In other words, we get $\Lambda^{r}[T]=() \star \Lambda^{1}[1] \cup \Omega[1]$. Thus, a lifting problem of shape

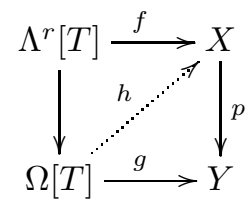

is equivalent to a lifting problem of shape

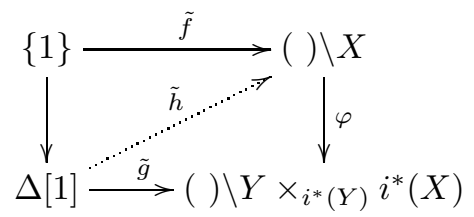

By virtue of Proposition 4.13, the map $\varphi$ is a left fibration, and, as left fibrations are stable by pullback and by composition, so is the projection of ()$\backslash Y \times i^{*}(Y) i^{*}(X)$ to $i^{*}(X)$. The image of $\tilde{g}$ by the latter is nothing but $f(r)$, and, as we know that left fibrations between $\infty$-categories are conservative (see [Joy02, Proposition 2.7]), the 1-cell $\tilde{g}$ is quasi-invertible in ()$\backslash Y \times_{i^{*}(Y)} i^{*}(X)$. We conclude the proof using Joy02, Propositions 2.4 and 2.7].

Proof of Theorem 4.2. Let $T$ be a tree with at least two vertices and a unary vertex $r$ at the root. There is a forest $\mathcal{T}=\left(T_{1}, \cdots, T_{k}\right), k \geqslant 0$, such that $T=\mathcal{T} \star 1$. By virtue of Lemma 4.15, we may assume that $\mathcal{T}$ is not the empty forest, or, equivalently, that $k \geqslant 1$. We will write $T^{\prime}=\mathcal{T} \star 0$. The trees $T$ and $T^{\prime}$ can be represented as follows.
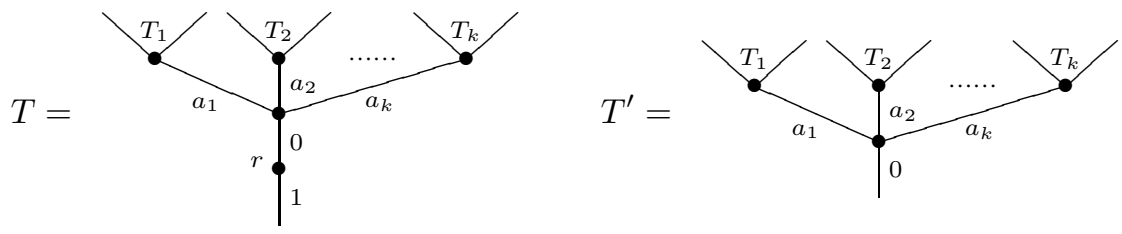

Given a dendroidal set $X$, a map $\Lambda^{r}[T] \longrightarrow X$ corresponds to a compatible family of maps of simplicial sets

$$
\{1\}=\Delta[0] \longrightarrow \partial_{A}\left(T^{\prime}\right) \backslash X
$$


indexed by the non-empty admissible subset of edges $A$ in $T^{\prime}$. This family corresponds to a map

$$
\Delta[0] \longrightarrow \varliminf_{A}^{\lim } \partial_{A}\left(T^{\prime}\right) \backslash X .
$$

By separating the case $A=\{0\}$ (the root edge of $T^{\prime}$ ) from the others, the map $\Lambda^{r}[T] \longrightarrow X$ corresponds to a commutative square of shape

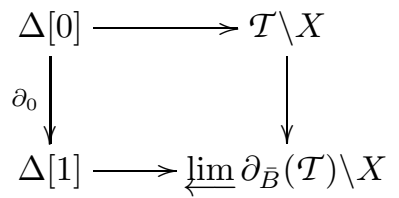

in which the limit $\varliminf_{\bar{B}} \partial_{\bar{B}}(\mathcal{T}) \backslash X$ is over the non-empty admissible subsets of edges $B$ in $T^{\prime}$ with $0 \notin B$, and $\bar{B}=\left(B \cap T_{1}, \ldots, B \cap T_{k}\right)$.

Consider from now on an inner Kan fibration between $\infty$-operads $p: X \longrightarrow Y$. Lifting problems of shape

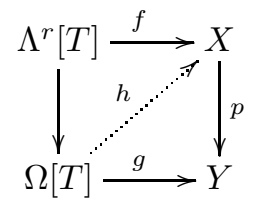

correspond to lifting problems

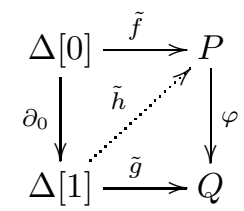

where $P=\mathcal{T} \backslash X$ and $Q=U \times_{W} V$, with

$$
U=\varliminf_{\bar{B}} \partial_{\bar{B}}(\mathcal{T}) \backslash X, \quad V=\mathcal{T} \backslash Y, \quad W=\varliminf_{\bar{B}} \partial_{\bar{B}}(\mathcal{T}) \backslash Y .
$$

Exactly like in the proof of Proposition 4.15, it now suffices to prove the following three statements:

(i) the map $\varphi: P \longrightarrow Q$ is a left fibration;

(ii) the simplicial set $Q$ is an $\infty$-category;

(iii) if $f(r)$ is weakly invertible in $X$, then the 1-cell $\tilde{g}$ is weakly invertible in $i^{*}(Q)$.

Note that, as left fibrations are conservative and are stable by pullback and composition, statements (ii) and (iii) will follow from the following two assertions:

(iv) the map $V \longrightarrow W$ is a left fibration;

(v) the map $U \longrightarrow i^{*}(X)$ is a left fibration.

But (iv) is a particular case of (i): just replace $p$ by the map from $Y$ to the terminal dendroidal set. It thus remains to prove (i) and (v).

4.15.1. Proof of (i). 
For $0 \leqslant i<n$, a lifting problem of the form

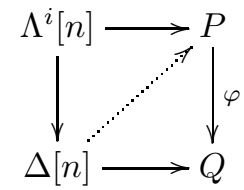

correspond to a lifting problem of the form

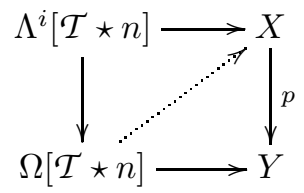

As $\Lambda^{i}[\mathcal{T} \star n]$ is an inner horn, (i) thus follows from the fact $p$ is an inner Kan fibration.

\subsubsection{Proof of (v).}

For $0 \leqslant i<n$, a lifting problem of the form

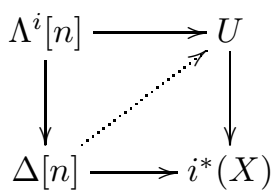

corresponds to a lifting problem of the form

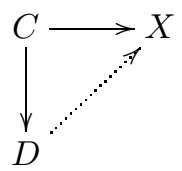

where the inclusion $C \longrightarrow D$ can be described as follows. The dendroidal set $D$ is the union of all the faces $\partial_{x}(\mathcal{T} \star n)$ given by contracting an inner edge or a root edge in one of the trees $T_{i}$, or by deleting a top vertex in the tree $\mathcal{T} \star n$. The dendroidal set $C$ is the union of the image of $\Omega[n] \longrightarrow \Omega[\mathcal{T} \star n]$ and all the 'codimension 2' faces of $\Omega[\mathcal{T} \star n]$ of shape $\partial_{j} \partial_{x}(\mathcal{T} \star n)$, where $\partial_{x}$ is as above, and $0 \leqslant j \leqslant n$ is distinct from $i$. It is now sufficient to check that the inclusion $C \longrightarrow D$ is an inner anodyne extension, which follows from a straightforward application of Lemma 4.10 .

\section{SUBDIVISION OF CYLINDERS}

5.1. Let $S$ be a tree with at least one vertex, and consider the tensor product $\Omega[S] \otimes \Delta[1]$. It has a subobject

$$
A_{0}=\partial \Omega[S] \otimes \Delta[1] \cup \Omega[S] \otimes\{1\}
$$

where $\{1\} \longrightarrow \Delta[1]$ is $\partial_{0}: \Delta[0] \longrightarrow \Delta[1]$. In this section, we will prove the following result.

Theorem 5.2. There exists a filtration of $\Omega[S] \otimes \Delta[1]$ of the form

$$
A_{0} \subset A_{1} \subset \cdots \subset A_{N-1} \subset A_{N}=\Omega[S] \otimes \Delta[1],
$$

where: 
(i) the inclusion $A_{i} \longrightarrow A_{i+1}$ is inner anodyne for $0 \leqslant i<N-1$;

(ii) the inclusion $A_{N-1} \longrightarrow A_{N}$ fits into a pushout of the form

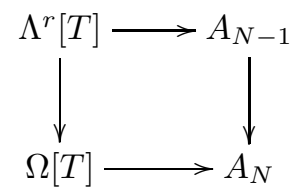

for a tree $T$ with at least two vertices and a unary vertex $r$ at the root;

(iii) the map

$$
\Delta[1] \longrightarrow \Lambda^{r}[T] \longrightarrow A_{N-1} \subset \Omega[S] \otimes \Delta[1]
$$

corresponding to the vertex $r$ in (ii) coincides with the inclusion

$$
\left\{e_{S}\right\} \otimes \Delta[1] \longrightarrow \Omega[S] \otimes \Delta[1]
$$

where $e_{S}$ is the edge at the root of the tree $S$.

5.3. The proof of Theorem 5.2 is in fact very similar to that of MW09, Proposition 9.2], stated here as Proposition 3.1. We recall from loc. cit. that, for any two trees $S$ and $T$, one can write

$$
\Omega[S] \otimes \Omega[T]=\bigcup_{i=1}^{N} \Omega\left[T_{i}\right],
$$

where $\Omega\left[T_{i}\right] \longrightarrow \Omega[S] \otimes \Omega[T]$ are 'percolation schemes'. Drawing vertices of $S$ as white, and those of $T$ as black, these percolation schemes are partially ordered in a natural way, starting with the tree obtained by stacking a copy of the black tree $T$ on top of each input edge of the white tree $S$, and ending with the tree obtained by stacking copies of $S$ on top of $T$. The intermediate trees are obtained by letting the black vertices of $T$ percolate through the white tree $S$, by successive 'moves' of the form

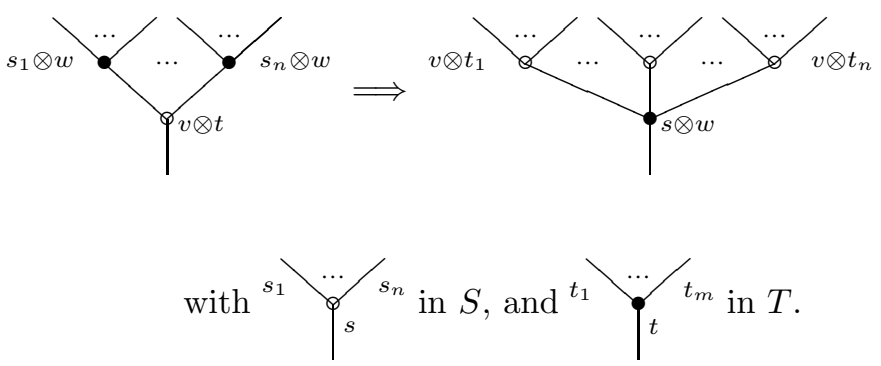

In the special case where $T=[1]$, the filtration referred to in Theorem 5.2 is given by

$$
A_{i}=A_{0} \cup \Omega\left[T_{1}\right] \cup \cdots \cup \Omega\left[T_{i}\right],
$$

where $T_{1}, \ldots, T_{N}$ is any linear order on the percolation schemes extending the natural partial order.

Remark 5.4. For any tree $S$ with at least one vertex, and root edge named $e_{S}$ ( $e$ for 'exit'), the last tree $T_{N}$ in the partial order of percolation schemes for $\Omega[S] \otimes \Delta[1]$ 
is of shape

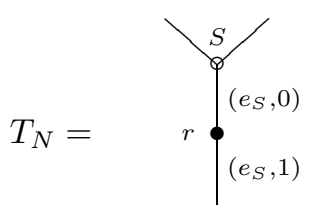

It always has a unique predecessor $T_{N-1}$ of the form

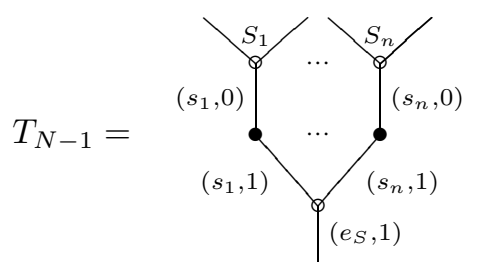

where $S$ is of the form $\left(S_{1}, \cdots, S_{n}\right) \star[0]$.

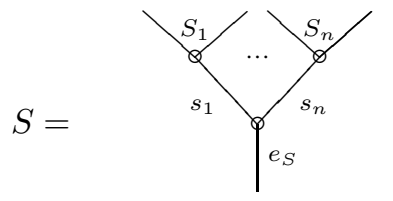

This observation already enables us to get

Proof of parts (ii) and (iii) of Theorem 5.2. Consider all the faces of $T_{N}$. For such a face $F \longrightarrow T_{N}$, there are three possibilities;

(a) it misses an $S$-colour entirely (i.e. there is an edge $s$ in $S$ so that neither $(s, 0)$ nor $(s, 1)$ are in $F$, so that $\Omega[F]$ factors through $\partial \Omega[S] \otimes \Delta[1]$;

(b) $F$ is given by contracting the edge $\left(e_{S}, 0\right)$, in which case $\Omega[F]$ factors through $\Omega\left[T_{N-1}\right]$ (since the face $F$ then coincides with the face of $T_{N-1}$ obtained by contracting $\left.\left(s_{1}, 1\right), \ldots,\left(s_{n}, 1\right)\right)$;

(c) $F$ is given by chopping off the edge $\left(e_{S}, 1\right)$ and the black vertex above it, i.e. $\Omega[F]=\Omega[S] \otimes\{0\}$. This face cannot factor through $A_{0}$, nor through any of the earlier percolation schemes since none of these has an edge coloured $\left(e_{S}, 0\right)$.

Thus, $\Omega\left[T_{N}\right] \cap A_{N-1}=\Lambda^{r}\left[T_{N}\right]$, where $r$ denotes the black vertex as pictured above. This shows that

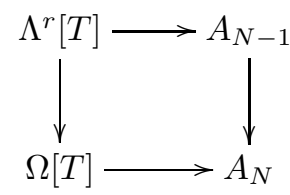

is a pushout, exactly as stated in part (ii) of Theorem 5.2. Moreover, the statement of part (iii) of Theorem 5.2 is obvious from the construction.

5.5. The proof of part (i) of Theorem 5.2 is more involved, but it is completely analogous to the proof of [MW09, Proposition 9.2]. The difference with the situation in loc. cit. is that, now, we are dealing with an inclusion of the form

$$
\partial \Omega[S] \otimes \Omega[T] \cup \Omega[S] \otimes \Lambda^{e}[T] \longrightarrow \Omega[S] \otimes \Omega[T],
$$


where $e$ is an outer edge of $T=i[1]$, whereas in loc. cit., we dealt with

$$
\Omega[S] \otimes \partial \Omega[T] \cup \Lambda^{e}[S] \otimes \Omega[T] \longrightarrow \Omega[S] \otimes \Omega[T],
$$

where $e$ is an inner edge of $S$. This forces us to look at different 'spines' and 'characteristic edges' compared to the ones in loc. cit. (notice also in this connection that although the tensor product is symmetric, the partial order on the percolation schemes is reversed).

The following lemma was also used (implicitly) in [MW09].

Lemma 5.6. Let $T_{i}$ and $T_{j}$ be two distinct percolation schemes for $\Omega[S] \otimes \Delta[1]$. Then

$$
\Omega\left[T_{i}\right] \cap \Omega\left[T_{j}\right] \subset \cup_{k} \Omega\left[T_{k}\right]
$$

as subobjects of $\Omega[S] \otimes \Delta[1]$, where the union ranges over all the percolation schemes $T_{k}$ which precede both $T_{i}$ and $T_{j}$ in the partial order.

Proof. Let $F$ be a common face of $\Omega\left[T_{i}\right]$ and $\Omega\left[T_{j}\right]$. If $T_{j} \leqslant T_{i}$ in the partial order, there is nothing to prove. Otherwise, we will give an algorithm for replacing $T_{j}$ by successively earlier percolation schemes,

$$
T_{j}=T_{j_{0}} \geqslant T_{j_{1}} \geqslant T_{j_{2}} \geqslant \cdots
$$

each having $F$ as a face, and eventually preceding $T_{i}$ in the partial order. As a first step, $T_{j}$ is obtained from an earlier percolation scheme $T_{j^{\prime}}$ by changing

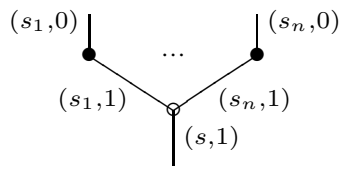

in $T_{j^{\prime}}$

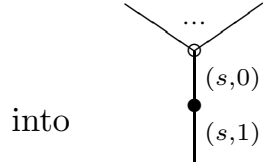

in $T_{j}$

If $F$ is also a face of $T_{j^{\prime}}$, we 'push up the black vertices' by replacing $T_{j}$ by $T_{j_{1}}=T_{j^{\prime}}$. If not, then the colour $(s, 0)$ must occur in $F$, hence in $T_{j}$ as well as in $T_{i}$. So the occurrence of $(s, 0)$ in $T_{j}$ is not the reason that $T_{j} \nless T_{i}$, and we put $T_{j_{1}}=T_{j}$. Treating all black vertices in this way, we can push them up if they occur below black vertices in $T_{i}$, until we eventually reach a percolation scheme $T_{j_{n}} \leqslant T_{j}$, still having $F$ as a face, for which $T_{j_{n}} \leqslant T_{i}$.

5.7. We return to the proof of Theorem 5.2. Consider the inclusion

$$
A_{k} \longrightarrow A_{k+1}=A_{k} \cup \Omega\left[T_{k+1}\right]
$$

for $k+1<N$. The percolation scheme $T_{k+1}$ will have at least one black vertex. Consider all the black vertices in $T_{k+1}$, and the corresponding faces of $T_{k+1}$ which are formed by paths from these black vertices to the root of $T_{k+1}$ :

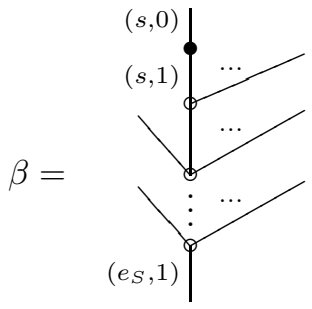


The face $\beta$ is the minimal external face which contains the given black vertex as well as the root edge. We call a face $\beta$ of $T_{k+1}$ of this form a spine in $T_{k+1}$. Notice that the vertex just above $\left(e_{S}, 1\right)$ is indeed white, as in the picture, because $k+1<N$. Notice also that the outer face of $\beta$ given by chopping off this vertex misses the colour $e_{S}$, hence belongs to $\partial \Omega[S] \otimes \Delta[1] \subset A_{0}$. Furthermore, the outer face of $\beta$ given by chopping off its black top vertex belongs to $\Omega[S] \otimes\{1\} \subset A_{0}$. Finally, all the inner faces of $\beta$ miss an $S$-color, hence factor through $\partial \Omega[S] \otimes \Delta[1]$, except possibly the one given by contracting the edge $(s, 1)$ near the top. However, if this last face $\partial_{(s, 1)}(\beta)$ of $\beta$ belongs to $A_{k}$, then some earlier $T_{i}, 1 \leqslant i \leqslant k$, contains the edge $(s, 0)$, hence all of $\beta$. Thus, either $\Omega[\beta]$ is contained in $A_{k}$, or we can adjoin it by an inner anodyne extension

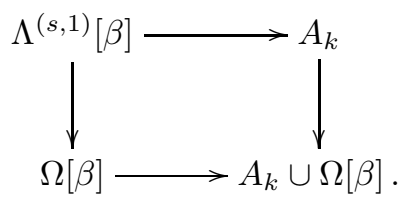

Such a spine $\beta$ is an example of an initial segment of $T_{k+1}$. Recall from [MW09. that a face $R \longrightarrow T_{k+1}$ is called an initial segment if it is obtained by successively chopping off top vertices. Our strategy will be to adjoin more initial segments of $T_{k+1}$ to $A_{k}$, starting with the spines. To this end, we need the following definition and lemma from [MW09, in which we use the notation $m(R) \subset \Omega\left[T_{k+1}\right]$ for the image of the map $\Omega[R] \longrightarrow \Omega\left[T_{k+1}\right]$ given by an initial segment $R$.

Definition 5.8 (MW09]). Let $R, Q_{1}, \ldots, Q_{p}$ be initial segments of $T_{k+1}$, and let $B=m\left(Q_{1}\right) \cup \cdots \cup m\left(Q_{p}\right)$. Suppose that, for every top face $F$ of $R$, we have $m(F) \subset A_{k} \cup B$. In this situation, an inner edge $\xi$ of $R$ is called characteristic with respect to $Q_{1}, \ldots, Q_{p}$ if, for any inner face $F$ of $R$, if $m(F / \xi)$ is contained in $A_{k} \cup B$, then so is $m(F)$ (where $F / \xi \longrightarrow F$ is the face obtained by contracting $\xi$ ).

Example 5.9. In any spine $\beta$ as in picture (5.7.2), the edge $\xi=(s, 1)$ is characteristic with respect to any family of initial segments.

Example 5.10. More generally, suppose $R$ is an initial segment of $T_{k+1}$ given by a spine $\beta$ expanded by one (or more) white vertices, say

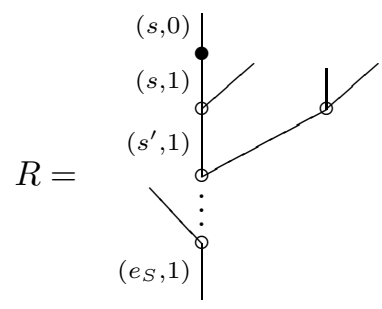

Then $\xi=(s, 1)$ is again characteristic with respect to any family $Q_{1}, \ldots, Q_{p}$. Indeed, if $R / \xi$ is a face of an initial segment $Q_{i}$, then so is $R$ itself; see [MW09, Remark 9.6 (iv)]. And if $R / \xi$ is a face of $T_{j}$ for a percolation scheme $T_{j}$, then $T_{j}$ 
either contains $R$, or looks like

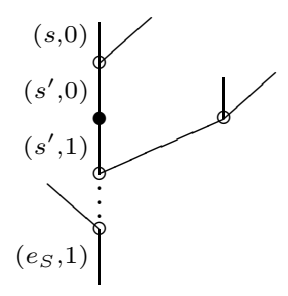

But, by Lemma 5.6, we can assume $T_{j}$ comes before $T_{k+1}$ in the partial order, so this is impossible. Finally, if $\Omega[R / \xi] \longrightarrow \Omega[S] \otimes \Delta[1]$ factors through $A_{0}$, then $R / \xi$ misses an $S$-colour, and hence so does $R$.

5.11. The proof of Theorem 5.2 (i) is based on a repeated use of arguments like the preceding one in Example 5.10. We quote the following lemma on characteristic edges from MW09.

Lemma 5.12 ([MW09, Lemma 9.7]). Let $R, Q_{1}, \ldots, Q_{p}$ be initial segments of $T_{k+1}$. Let $B=m\left(Q_{1}\right) \cup \cdots \cup m\left(Q_{p}\right)$, and suppose each top face of $R$ has the property that $m(F)$ is contained in $A_{k} \cup B$. If $R$ possesses a characteristic edge with respect to $Q_{1}, \ldots, Q_{p}$, then the inclusion

$$
A_{k} \cup B \longrightarrow A_{k} \cup B \cup m(R)
$$

of subobjects of $\Omega[S] \otimes \Delta[1]$ is inner anodyne.

Lemma 5.13. Let $R, Q_{1}, \ldots, Q_{p}$ be initial segments of $T_{k+1}$, satisfying condition (i) in Definition 5.8, and let $\beta$ be a spine in $R$. Then the edge $\xi=(s, 1)$ immediately below the black vertex on the spine is a characteristic edge for $R$.

Hint for a proof. This is proved exactly as Example 5.10, cf. also MW09, Lemma 9.8].

5.14. Using the characteristic edges from Lemma 5.13 , one can now copy the proof of [MW09, Lemma 9.9], repeated below as Lemma [5.15] verbatim. This proof is by induction on $l$, and describes a precise strategy for adjoining more and/or larger initial segments of $T_{k+1}$ to $A_{k}$.

Lemma 5.15. Fix $l \leqslant 0$, and let $Q_{1}, \ldots, Q_{p}$ be a family of initial segments in $T_{k+1}$, each containing at least one spine, and at most $l$ spines (so, necessarily, $p=0$ if $l=0)$. Let $R_{1}, \ldots, R_{q}$ be initial segments which each contain exactly $l+1$ spines. Then the inclusion $A_{k} \longrightarrow A_{k} \cup B \cup C$ is inner anodyne, where $B=m\left(Q_{1}\right) \cup \cdots \cup m\left(Q_{p}\right)$ and $C=m\left(R_{1}\right) \cup \cdots \cup m\left(R_{q}\right)$.

5.16. This strategy terminates when one arrives at the number $l$ of all spines in $T_{k+1}$. Indeed, for this $l$ and $p=0, q=1$, Lemma 5.15 states for $R_{1}=T_{k+1}$ that $A_{k} \longrightarrow A_{k+1}$ is inner anodyne, as asserted in Theorem 5.2 (i). This completes the proof of Theorem 5.2 .

\section{6. $\infty$-OPERADS AS FIBRANT OBJECTS}

6.1. The aim of this section is to characterize $\infty$-operads as the fibrant objects of the model category structure on the the category of dendroidal sets given by Proposition 3.12. This characterization is stated in Theorem 6.10 below. 
Given an $\infty$-category $X$, we denote by $k(X)$ the maximal Kan complex contained in $X$; see [Joy02, Corollary 1.5].

Recall that, given two dendroidal sets $A$ and $X$, we write

$$
\operatorname{fom}(A, X)=i^{*} \mathcal{H o m}(A, X) \text {. }
$$

Note that, by virtue of Proposition 3.1 if $X$ is an $\infty$-operad, and if $A$ is normal, then $\operatorname{Hom}(A, X)$ is an $\infty$-operad, so that $\operatorname{fom}(A, X)$ is an $\infty$-category.

For an $\infty$-operad $X$ and a simplicial set $K$, we will write $X^{(K)}$ for the subcomplex of $\mathcal{H o m}\left(i_{!}(K), X\right)$ which consists of dendrices

$$
a: \Omega[T] \times i_{!}(K) \longrightarrow X
$$

such that, for any 0-cell $u$ in $T$, the induced map

$$
a_{u}: K \longrightarrow i^{*}(X)
$$

factors through $k\left(i^{*}(X)\right)$ (i.e. all the 1-cells in the image of $a_{u}$ are weakly invertible in $\left.i^{*}(X)\right)$.

For an $\infty$-operad $X$ and a normal dendroidal set $A$, we will write $k(A, X)$ for the subcomplex of fom $(A, X)$ which consists of maps

$$
u: A \otimes i_{!}(\Delta[n]) \longrightarrow X
$$

such that, for all vertices $a$ of $A$ (i.e. maps $a: \eta \longrightarrow A$ ), the induced map

$$
u_{a}: \Delta[n] \longrightarrow i^{*}(X)
$$

factors through $k\left(i^{*}(X)\right)$. So, by definition, for any normal dendroidal set $A$, any simplicial set $K$, and any $\infty$-operad $X$, there is a natural bijection:

$$
\operatorname{Hom}_{s \mathcal{S} e t}(K, k(A, X)) \simeq \operatorname{Hom}_{\text {dSet }}\left(A, X^{(K)}\right) .
$$

Remark 6.2. The simplicial set $k(A, X)$ is by definition the $\infty$-category of objectwise weakly invertible 1-cells in $\operatorname{fom}(A, X)$. We can reformulate the definition of $k(A, X)$ as follows (still with $A$ normal and $X$ an $\infty$-operad). Define

$$
\mathrm{Ob} A=\coprod_{A_{0}} \eta \text {. }
$$

We have a unique monomorphism $i: \mathrm{Ob} A \longrightarrow A$ which is the identity on 0-cells. As $A$ is normal, $i$ is a normal monomorphism. We also have

$$
k(\mathrm{Ob} A, X)=k(\operatorname{hom}(\mathrm{Ob} A, X))=\prod_{A_{0}} k\left(i^{*} X\right),
$$

and $k(A, X)$ fits by definition in the following pullback square.

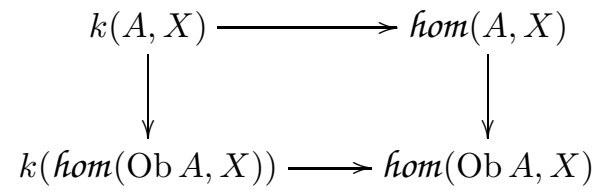

In particular, the projection of $k(A, X)$ on $k(\mathrm{Ob} A, X)$ is an inner Kan fibration, and as the latter is a Kan complex, this shows that $k(A, X)$ is an $\infty$-category. One of the key results of this section asserts that $k(A, X)$ is a Kan complex as well, which can be reformulated by saying that the inclusion $k(\operatorname{fom}(A, X)) \subset k(A, X)$ is in fact an equality. In other words, a map in the $\infty$-category from $(A, X)$ is weakly invertible if and only it is objectwise weakly invertible; see Corollary 6.8, 
6.3. Before stating the next theorem, we recall that, for a morphism between $\infty$ categories $f: X \longrightarrow Y$, the induced map $\tau(f): \tau(X) \longrightarrow \tau(Y)$ is a categorical fibration if and only if the map

$$
e v_{1}: X^{(\Delta[1])} \longrightarrow Y^{(\Delta[1])} \times_{Y} X
$$

induced by evaluating at 1 (i.e. by the inclusion $\{1\} \longrightarrow \Delta[1]$ ) has the right lifting property with respect to $\partial \Delta[0] \longrightarrow \Delta[0]$; see Joy02, Proposition 2.4].

Theorem 6.4. Let $p: X \longrightarrow Y$ be an inner Kan fibration between $\infty$-operads. The map ev $: X^{(\Delta[1])} \longrightarrow Y^{(\Delta[1])} \times_{Y} X$ has the right lifting property with respect to inclusions $\partial \Omega[S] \longrightarrow \Omega[S]$ for any tree $S$ with at least one vertex. Consequently, the functor $\tau i^{*}(p)$ is a categorical fibration if and only if the evaluation at 1 map $X^{(\Delta[1])} \longrightarrow Y^{(\Delta[1])} \times_{Y} X$ is a trivial fibration of dendroidal sets.

Proof. Consider a tree $S$ with at least one vertex and a solid commutative square

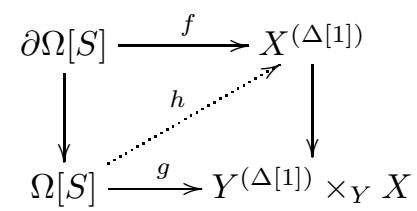

We want to prove the existence of a diagonal filling $h$. This corresponds by adjunction to a filling $\tilde{h}$ in the following commutative square

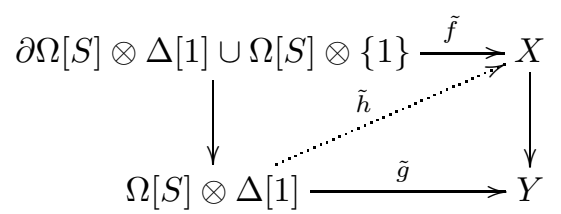

(as the inclusion of $\partial \Omega[S]$ in $\Omega[S]$ is bijective on objects, and as the restriction of $\tilde{h}$ to $\partial \Omega[S] \otimes \Delta[1] \cup \Omega[S] \otimes\{1\}$ coincides with $\tilde{f}$, the map $\Omega[S] \longrightarrow X^{\Delta[1]}$ corresponding to a filling $\tilde{h}$ will automatically factor through $\left.X^{(\Delta[1])}\right)$.

Consider the filtration

$$
\partial \Omega[S] \otimes \Delta[1] \cup \Omega[S] \otimes\{1\}=A_{0} \subset A_{1} \subset \cdots \subset A_{N-1} \subset A_{N}=\Omega[S] \otimes \Delta[1]
$$

given by Theorem 5.2. As the map $X \longrightarrow Y$ is an inner Kan fibration, using Theorem 5.2 (i), it is sufficient to find a filling in a solid commutative diagram of shape

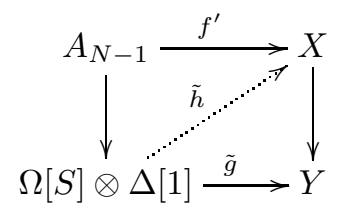

in which the restriction of $f^{\prime}$ to $\partial \Omega[S] \otimes \Delta[1] \cup \Omega[S] \otimes\{1\}$ coincides with $\tilde{f}$. By virtue of Theorem 5.2 (ii), it is even sufficient to find a filling $k$ in a solid commutative 
diagram of shape

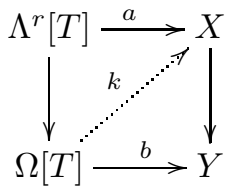

in which $T$ is a tree with unary vertex $r$ at the root, and $a$ is the restriction of $f^{\prime}$ to $\Lambda^{r}[T] \subset A_{N-1}$. Furthermore, by Theorem 5.2 (iii), we may assume that $a(r)$ is weakly invertible in $i^{*}(X)$. Thus, the existence of the filling $k$ is ensured by Theorem 4.2.

The last assertion of the theorem follows from 6.3

Lemma 6.5. Any left fibration between Kan complexes is a Kan fibration.

Proof. This follows from [Joy02, Theorem 2.2 and Proposition 2.7].

Lemma 6.6. A morphism of simplicial sets $X \longrightarrow Y$ is a left (resp. right) fibration if and only it has the right lifting property with respect to maps of shape

$$
\partial \Delta[n] \times \Delta[1] \cup \Delta[n] \times\{e\} \longrightarrow \Delta[n] \times \Delta[1]
$$

for $e=1$ (resp. for $e=0$ ) and $n \geqslant 0$.

Proof. The map $\partial \Delta[n] \times \Delta[1] \cup \Delta[n] \times\{0\} \longrightarrow \Delta[n] \times \Delta[1]$ is obtained as a finite composition of pushouts of horns of shape $\Lambda^{k}[n+1] \longrightarrow \Delta[n+1]$ with $0 \leqslant k<n+1$; see (the dual version of) GZ67, Chapter IV, 2.1.1].

Conversely, the inclusion map $\Lambda^{k}[n] \longrightarrow \Delta[n], 0 \leqslant k<n$, is a retract of the map $\Lambda^{k}[n] \times \Delta[1] \cup \Delta[n] \times\{0\} \longrightarrow \Delta[n] \times \Delta[1]$; see [GZ67, Chapter IV, 2.1.3].

We deduce easily from this that a morphism of simplicial sets $X \longrightarrow Y$ is a right fibration if and only if the evaluation at 0 map $X^{\Delta[1]} \longrightarrow Y^{\Delta[1]} \times_{Y} X$ is a trivial fibration (i.e. has the right lifting property with respect to monomorphisms). The case of left fibrations follows by duality.

Proposition 6.7. Let $p: X \longrightarrow Y$ be an inner Kan fibration between $\infty$-operads. If $\tau i^{*}(p)$ is a categorical fibration, then, for any monomorphism between normal dendroidal sets $A \longrightarrow B$, the map

$$
k(B, X) \longrightarrow k(B, Y) \times_{k(A, Y)} k(A, X)
$$

is a Kan fibration between Kan complexes.

Proof. The functor $i_{!}:$sSet $\longrightarrow d$ Set being symmetric monoidal and preserving inner anodyne extensions, Proposition 3.1 implies that the map

$$
\operatorname{hom}(B, X) \longrightarrow \operatorname{fom}(B, Y) \times \operatorname{hom}(A, Y) \operatorname{from}(A, X)
$$

is an inner Kan fibration between $\infty$-categories. This implies the map

$$
k(B, X) \longrightarrow k(B, Y) \times_{k(A, Y)} k(A, X)
$$

is an inner Kan fibration between $\infty$-categories. 
We claim that this map has the right lifting property with respect to the inclusion $\{1\} \longrightarrow \Delta[1]$. Using the identification (6.1.1), we see that lifting problems of shape

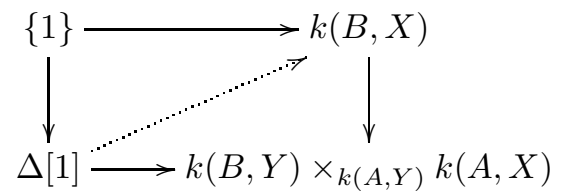

correspond to lifting problems of shape

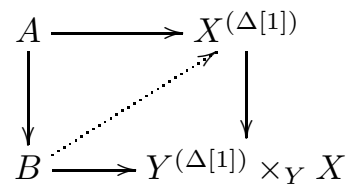

so that our claim follows from Theorem 6.4 .

More generally, the map $k(B, X) \longrightarrow k(B, Y) \times_{k(A, Y)} k(A, X)$ has the right lifting property with respect to maps of shape

$$
\partial \Delta[n] \times \Delta[1] \cup \Delta[n] \times\{1\} \longrightarrow \Delta[n] \times \Delta[1], \quad n \geqslant 0 .
$$

We have just checked it above in the case where $n=0$, so that it remains to prove the case where $n>0$. Consider a lifting problem of shape

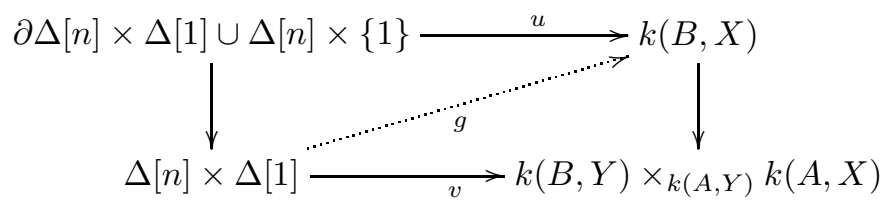

This lifting problem gives rise to a lifting problem of shape

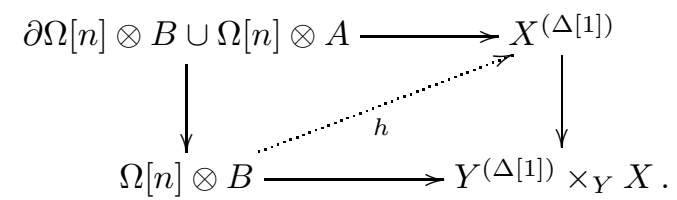

The existence of the lifting $h$ is provided again by Theorem 6.4, The map $h$ defines a map

$$
l: i_{!}(\Delta[n] \times \Delta[1]) \otimes B \longrightarrow X .
$$

As a consequence, it is sufficient to check that, for every non-degenerate $m$-simplex $\delta: \Delta[m] \longrightarrow \Delta[n] \times \Delta[1], m \geqslant 1$, and for any object $b: \eta \longrightarrow B$, the map $\left(l i_{!}(\delta)\right)_{b}: \Delta[m] \longrightarrow i^{*}(X)$ factors through $k\left(i^{*}(X)\right)$. Using the '2 out of 3 property' for weakly invertible 1 -cells in $i^{*}(X)$, we can assume that $m=1$. But then, as $n>0$, using again the ' 2 out of 3 property' for weakly invertible 1-cells, we may assume that $\delta$ factors through $\partial \Delta[n] \times \Delta[1]$, which implies then that $\left(l \circ i_{!}(\delta)\right) \otimes$ $1_{B}$ factors through the subcomplex $i_{!}(\partial \Delta[n] \times \Delta[1]) \otimes B$ : the required property thus follows from the fact that the restriction of the transpose of $h$ to the object $i_{!}(\Delta[n] \times \Delta[1] \cup \partial \Delta[n] \times\{1\}) \otimes B$ corresponds to the map $u$ in (6.7.4).

By virtue of Lemma 6.6 the map $k(B, X) \longrightarrow k(B, Y) \times_{k(A, Y)} k(A, X)$ is a left fibration, hence, by Joy02, Proposition 2.7], is conservative. By applying [Joy02, Corollary 1.4], we deduce, from the case where $A=\varnothing$ and $Y$ is the terminal 
dendroidal set, that $k(B, X)$ is a Kan complex for any normal dendroidal set $B$ and any $\infty$-operad $X$. As any left fibration between Kan complexes is a Kan fibration (Lemma 6.5), the maps $k(B, X) \longrightarrow k(A, X)$ are thus Kan fibrations between Kan complexes for any monomorphisms between normal dendroidal sets $A \longrightarrow B$ and any $\infty$-operad $X$. As a consequence, Kan fibrations being stable by pullback, we see that the fiber product $k(B, Y) \times_{k(A, Y)} k(A, X)$ is a Kan complex. Using again Lemma 6.5, we conclude that $k(B, X) \longrightarrow k(B, Y) \times_{k(A, Y)} k(A, X)$ is a Kan fibration between Kan complexes.

Corollary 6.8. For any normal dendroidal set $A$ and any $\infty$-operad $X$, we have

$$
k(\operatorname{fom}(A, X))=k(A, X) .
$$

For any inner Kan fibration between $\infty$-operads $p: X \longrightarrow Y$ such that $\tau i^{*}(p)$ is a categorical fibration, and for any monomorphism between normal dendroidal sets $A \longrightarrow B$, we have

$$
k\left(\operatorname{from}(B, Y) \times_{\operatorname{hom}(A, Y)} \operatorname{fom}(A, X)\right)=k(B, Y) \times_{k(A, Y)} k(A, X) .
$$

Proof. If $A$ is normal, then, for any operad $X, k(A, X)$ is a Kan complex which contains $k(\operatorname{fom}(A, X))$. As $k(\operatorname{from}(A, X))$ is the maximal sub Kan complex contained in the $\infty$-category from $(A, X)$, this proves the first assertion. The second assertion is proved similarly.

Corollary 6.9. Let $p: X \longrightarrow Y$ be an inner Kan fibration between $\infty$-operads. If $\tau i^{*}(p)$ is a categorical fibration, then, for any anodyne extension of simplicial sets $K \longrightarrow L$, the map

$$
X^{(L)} \longrightarrow Y^{(L)} \times_{Y^{(K)}} X^{(K)}
$$

is a trivial fibration of dendroidal sets.

Proof. This follows from Proposition 6.7 and from the natural identification (6.1.1).

Theorem 6.10. A dendroidal set is $J$-fibrant if and only if it is an $\infty$-operad. An inner Kan fibration between $\infty$-operads $p: X \longrightarrow Y$ is a J-fibration (i.e. a fibration for the model category structure of Proposition [3.12) if and only if $\tau i^{*}(p)$ is a categorical fibration.

Proof. Let $p: X \longrightarrow Y$ be inner Kan fibration between $\infty$-operads. We have to prove that, for $e=0,1$, the anodyne extension $\{e\} \longrightarrow J$ induces a trivial fibration of dendroidal sets

$$
X^{J_{d}} \longrightarrow Y^{J_{d}} \times_{Y} X
$$

if and only if $\tau i^{*}(p)$ is a categorical fibration. But, for any $\infty$-operad $Z$, we clearly have $Z^{J_{d}}=Z^{(J)}$ and $Z=Z^{(\{e\})}$. Hence, by virtue of Corollary 6.9, if $\tau i^{*}(p)$ is a categorical fibration, then $p$ is a $J$-fibration. The converse is a direct consequence of [Joy02, Corollary 1.6].

Corollary 6.11. The class of weak operadic equivalences is the smallest class of maps of dendroidal sets $\mathrm{W}$ which satisfies the following three properties.

(a) (' 2 out 3 property') In any commutative triangle, if two maps are in $\mathrm{W}$, then so is the third.

(b) Any inner anodyne extension is in $\mathrm{W}$.

(c) Any trivial fibration between $\infty$-operads is in $\mathrm{W}$. 
Proof. Consider a class of maps W satisfying conditions (a), (b) and (c) above. We want to prove that any weak operadic equivalence is in $\mathrm{W}$.

Let $f: A \longrightarrow B$ be a morphism of dendroidal sets. Using the small object argument applied to the set of inner horns, we can see there exists a commutative square

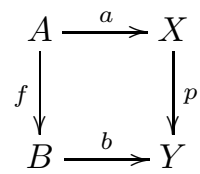

in which the maps $a$ and $b$ are inner anodyne extensions, and $X$ and $Y$ are $\infty$ operads. It is clear that $f$ is a weak operadic equivalence (resp. is in $\mathrm{W}$ ) if and only $p$ has the same property. Hence it is sufficient to prove that any weak operadic equivalence between $\infty$-operads is in W. As any trivial fibration between $\infty$-operads is in $\mathrm{W}$ by assumption, and as $\infty$-operads are the fibrant objects of a model category, this corollary follows from Ken Brown's Lemma Hov99, Lemma 1.1.12].

6.12. We will write $C_{n}$ for the corolla with $n+1$ edges,

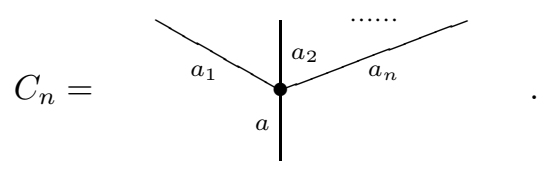

Let $X$ be an $\infty$-operad. Given an $(n+1)$-tuple of 0 -cells $\left(x_{1}, \ldots, x_{n}, x\right)$ in $X$, the space of maps $X\left(x_{1}, \ldots, x_{n} ; x\right)$ is obtained by the pullback below, in which the map $p$ is the map induced by the inclusion $\eta \amalg \cdots \amalg \eta \longrightarrow \Omega\left[C_{n}\right]$ (with $n+1$ copies of $\eta$, corresponding to the $n+1$ objects $\left(a_{1}, \ldots, a_{n}, a\right)$ of $\left.C_{n}\right)$.

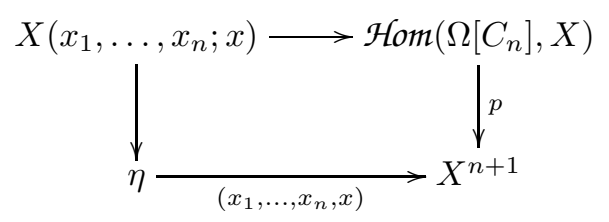

Using the identification $s \mathcal{S e t}=d \operatorname{Set} / \eta$, we shall consider $X\left(x_{1}, \ldots, x_{n} ; x\right)$ as a simplicial set.

Proposition 6.13. The simplicial set $X\left(x_{1}, \ldots, x_{n} ; x\right)$ is a Kan complex.

Proof. The first assertion of Corollary 6.8 for $A=\Omega\left[C_{n}\right]$ can be reinterpreted by saying we have the pullback square below (see Remark 6.2).

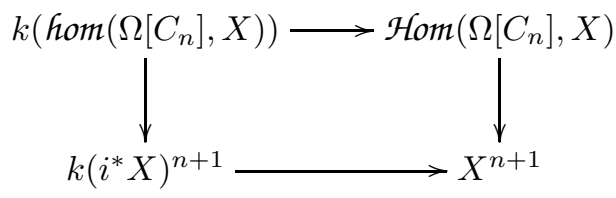


As the terminal simplicial set $\eta$ is certainly a Kan complex, it thus follows from the construction of $X\left(x_{1}, \ldots, x_{n} ; x\right)$ that we have a pullback square

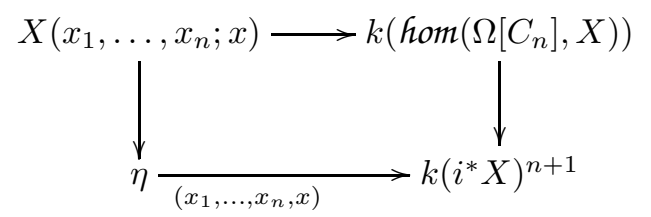

in which the right vertical map in this diagram is a Kan fibration (by Proposition 6.7. applied for $A=\eta \amalg \cdots \amalg \eta$ and $\left.B=\Omega\left[C_{n}\right]\right)$. The stability of Kan fibrations by pullback achieves the proof.

Proposition 6.14. There is a canonical bijection

$$
\pi_{0}\left(X\left(x_{1}, \ldots, x_{n} ; x\right)\right) \simeq \tau_{d}(X)\left(x_{1}, \ldots, x_{n} ; x\right) .
$$

Proof. We will use the explicit description of $\tau_{d}(X)$ given by MW09, Lemma 6.4 and Proposition 6.6]. The unit map $X \longrightarrow N_{d} \tau_{d}(X)$ induces a map

$$
X\left(x_{1}, \ldots, x_{n} ; x\right) \longrightarrow\left(N_{d} \tau_{d}(X)\right)\left(x_{1}, \ldots, x_{n} ; x\right) .
$$

It is easily seen that $\left(N_{d} \tau_{d}(X)\right)\left(x_{1}, \ldots, x_{n} ; x\right)$ is the discrete simplicial set associated to $\tau_{d}(X)\left(x_{1}, \ldots, x_{n} ; x\right)$, so that we get a surjective map

$$
\pi_{0}\left(X\left(x_{1}, \ldots, x_{n} ; x\right)\right) \longrightarrow \tau_{d}(X)\left(x_{1}, \ldots, x_{n} ; x\right) .
$$

Using the explicit description of $\tau_{d}(X)$ given by MW09, Lemma 6.4 and Proposition 6.6], it is now sufficient to prove that, if $f$ and $g$ are two 0 -simplices of $X\left(x_{1}, \ldots, x_{n} ; x\right)$ which are homotopic along the edge 0 in the sense of [MW09, Definition 6.2], then they belong to the same connected component. But then, $f$ and $g$ define two objects of $\tau\left(\operatorname{fom}\left(\Omega\left[C_{n}\right], X\right)\right)$ which are isomorphic, which can be expressed by the existence of a map

$$
h: \Delta[1] \longrightarrow k\left(\operatorname{from}\left(\Omega\left[C_{n}\right], X\right)\right)
$$

which connect $f$ and $g$. Using that $k\left(\operatorname{from}\left(\Omega\left[C_{n}\right], X\right)\right) \longrightarrow i^{*}(X)^{n+1}$ is a Kan fibration between Kan complexes, we can see by a path lifting argument that such a map $h$ is homotopic under $\partial \Delta[1]$ to a map $\Delta[1] \longrightarrow X\left(x_{1}, \ldots, x_{n} ; x\right)$ which connects $f$ and $g$.

Lemma 6.15. Let $X \longrightarrow Y$ be a trivial fibration between $\infty$-operads. Then, for any $(n+1)$-tuple of 0 -cells $\left(x_{1}, \ldots, x_{n}, x\right)$ in $X$, the induced map

$$
X\left(x_{1}, \ldots, x_{n} ; x\right) \longrightarrow Y\left(f\left(x_{1}\right), \ldots, f\left(x_{n}\right) ; f(x)\right)
$$

is a trivial fibration of simplicial sets.

Proof. We know that the map

$$
\operatorname{Hom}\left(\Omega\left[C_{n}\right], X\right) \longrightarrow \mathcal{H o m}\left(\Omega\left[C_{n}\right], Y\right) \times_{Y^{n+1}} X^{n+1}
$$

is a trivial fibration (this follows from Proposition 1.9 by adjunction). As we have a pullback of shape

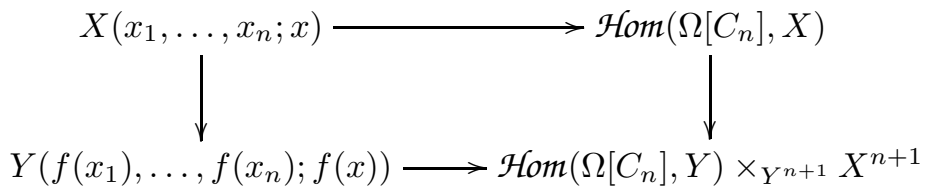


this proves the lemma.

Proposition 6.16. The functor $\tau_{d}:$ dSet $\longrightarrow$ Operad sends weak operadic equivalences to equivalences of operads.

Proof. We know that $\tau_{d}$ sends inner horn inclusions to isomorphisms of operads (this follows from [MW09, Theorem 6.1] by the Yoneda Lemma). As $\tau_{d}$ preserves colimits, we deduce that $\tau_{d}$ sends inner anodyne extensions to isomorphisms of operads. By virtue of Corollary 6.11, it is thus sufficient to prove that $\tau_{d}$ sends trivial fibrations between $\infty$-operads to equivalences of operads. Let $f: X \longrightarrow Y$ be a trivial fibration between $\infty$-operads. By virtue of Proposition 6.14 and of Lemma 6.15, we see that $\tau_{d}(f)$ is fully faithful. As $f$ is obviously surjective on 0 -cells, $\tau_{d}(f)$ has to be an equivalence of operads.

Corollary 6.17. The adjunction $\tau_{d}:$ dSet $\rightleftarrows$ Operad $: N_{d}$ is a Quillen pair. Moreover, the two functors $\tau_{d}$ and $N_{d}$ both preserve weak equivalences. In particular, a morphism of operads is an equivalence of operads if and only if its dendroidal nerve is a weak operadic equivalence.

Proof. The functor $\tau_{d}$ preserves cofibrations, so that this is a direct consequence of Proposition 6.16. Note that any operad is fibrant, so that the dendroidal nerve functor $N_{d}$ preserves weak equivalences. Hence the last assertion comes from the fact $N_{d}$ is fully faithful and $\tau_{d}$ preserves weak equivalences.

Remark 6.18. Theorem 6.10 also asserts that the functor $\tau_{d}$ preserves fibrations between $\infty$-operads.

6.19. If $A$ is a normal dendroidal set, and if $X$ is an $\infty$-operad, we have

$$
\operatorname{Hom}_{\mathbf{H o}(d S e t)}(A, X)=[A, X] \simeq \pi_{0}(k(\operatorname{fom}(A, X))) .
$$

Indeed, $J \otimes A$ is a cylinder of $A$, and morphisms

$$
J \otimes A \longrightarrow X
$$

correspond to morphisms

$$
J \longrightarrow k(\operatorname{fom}(A, X))
$$

so that this formula follows from the fact $X$ is $J$-fibrant. The next statement is a reformulation of (6.19.1).

Proposition 6.20. Let $A$ be a normal dendroidal set, and $X$ an $\infty$-operad. The set $[A, X]=\operatorname{Hom}_{\mathbf{H o}(d S e t)}(A, X)$ can be canonically identified with the set of isomorphism classes of objects in the category $\tau$ hom $(A, X)$ (which is also the category underlying $\left.\tau_{d}(\mathcal{H o m}(A, X))\right)$.

Proof. This proposition is a direct application of the explicit description of the operad $\tau_{d}(\mathcal{H}$ om $(A, X))$ given by [MW09, Proposition 6.6] and of Corollary 6.9.

\section{Appendices}

\section{A. Grafting orders onto trees}

The main goal of the technical sections 4 and 5 was to deduce Theorem 6.10, and from it, Corollary 6.9. There is an asymmetry in this approach, in that Theorem 
6.10 was only proved for evaluation at one of the end points, and the symmetry was established in Corollary 6.9 by using the theory of left fibrations between simplicial sets.

In these two appendices, we will prove the analogs of Theorems 4.2 and 5.2 from which one can deduce directly the symmetric version of Theorem 6.10 (for evaluation at 0 ). These two appendices can also be used as an alternative approach to the results in Section 6. Moreover, they are of interest by themselves, as they form the basis of a theory of right fibrations of dendroidal sets.

However, since the left-right duality for simplicial sets does not extend to dendroidal sets, the results of these appendices cannot be deduced from their analogs proved earlier.

We begin by studying the analog of Theorem 4.2 (see Theorem A.7 below).

A.1. Let $T$ be a tree endowed with an input edge (leaf) $e$.

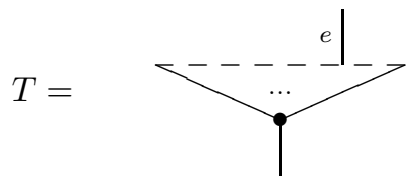

Given an integer $n \geqslant 0$, we define the tree $n \star_{e} T$ as the tree obtained by joining the $n$-simplex to the edge $e$ by a new vertex $v$.

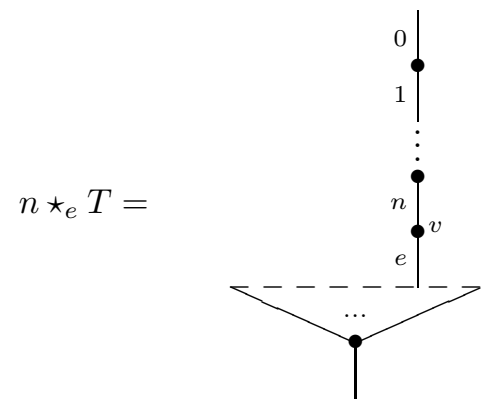

This defines a unique functor

$$
\Delta \longrightarrow \Omega, \quad[n] \longmapsto n \star_{e} T
$$

such that the obvious inclusions $i[n] \longrightarrow n \star_{e} T$ are functorial. We thus get a functor

$$
(-) \star_{e} T: \Delta \longrightarrow T / d S e t
$$

(where $T / d S e t$ denotes the category of dendroidal sets under $\Omega[T]$ ). By Kan extension, we obtain a colimit preserving functor

$$
(-) \star_{e} T: \text { sSet } \longrightarrow T / d \text { Set } .
$$

We have $\Delta[n] \star_{e} T=\Omega\left[n \star_{e} T\right]$. The functor A.1.5 has a right adjoint

$$
(-) / e^{T: T / d S e t} \longrightarrow \text { sSet } .
$$

Remark A.2 (Functoriality in $T$ ). We shall say that a face map $R \longrightarrow T$ is $e$ admissible if it does not factor through the external face map which chops off $e$. For such a face $R \longrightarrow T, e$ is also a leaf of $R$, and there are natural maps

$$
n \star_{e} R \longrightarrow n \star_{e} T \text {. }
$$


Thus, we obtain, for each simplicial set $K$, and each dendroidal set $X$ under $T$ (i.e. under $\Omega[T]$ ), natural maps

$$
K \star_{e} R \longrightarrow K \star_{e} T
$$

and

$$
X /{ }_{e} T \longrightarrow X /{ }_{e} R
$$

Similarly, the inclusions $\Omega[n] \longrightarrow \Delta[n] \star_{e} T$ induce a projection

$$
X / e^{T} \longrightarrow i^{*}(X)
$$

for any dendroidal set $X$ under $T$.

A.3. Let $0<i \leqslant n$ be integers. Let $\left\{R_{1}, \ldots, R_{t}\right\}, t \geqslant 1$, be a finite family of $e$-admissible faces of $T$, and define

$$
C \subset D \subset \Omega\left[n \star_{e} T\right]
$$

by

$$
C=\left(\bigcup_{s=1}^{t} \Lambda^{i}[n] \star_{e} R_{s}\right) \cup \Omega[n] \text { and } D=\bigcup_{s=1}^{t} \Delta[n] \star_{e} R_{s},
$$

where $\Omega[n]$ is seen as a subcomplex of $\Omega\left[n \star_{e} T\right]$ through the canonical embedding.

Lemma A.4. Under the assumptions of A.3, the map $C \longrightarrow D$ is an inner anodyne extension.

Proof. For $p \geqslant 1$, write $\mathcal{F}_{p}$ for the set of faces $F$ of $\Omega\left[n \star_{e} T\right]$ which belong to $D$ but not to $C$, and which are of the form $F=\Omega\left[n \star_{e} R\right]$ for an $e$-admissible face $R$ of $T$ with exactly $p$ edges. Define a filtration

$$
C=C_{0} \subset C_{1} \subset \ldots \subset C_{p} \subset \ldots \subset D
$$

by

$$
C_{p}=C_{p-1} \cup \bigcup_{F \in \mathcal{F}_{p}} F, \quad p \geqslant 1 .
$$

We have $D=C_{p}$ for $p$ big enough, and it is sufficient to prove that the inclusions $C_{p-1} \longrightarrow C_{p}$ are inner anodyne for $p \geqslant 1$. If $F$ and $F^{\prime}$ are in $\mathcal{F}_{p}$, then $F \cap F^{\prime}$ is in $C_{p-1}$. Moreover, if $F=\Omega\left[n \star_{e} R\right]$ for an $e$-admissible face $R$ of $T$, then we have

$$
F \cap C_{p-1}=\Lambda^{i}\left[n \star_{e} R\right],
$$

which is an inner horn. Hence we can describe the inclusion $C_{p-1} \longrightarrow C_{p}$ as a finite composition of pushouts by inner horn inclusions of shape $F \cap C_{p-1} \longrightarrow F$ for $F \in \mathcal{F}_{p}$.

Proposition A.5. Let $0<i \leqslant n$ be integers. The inclusion

$$
\left(\Lambda^{i}[n] \star_{e} T\right) \cup \Omega[n] \longrightarrow \Omega\left[n \star_{e} T\right]
$$

is an inner anodyne extension.

Proof. Apply Lemma A.4.

Proposition A.6. For any inner Kan fibration $p: X \longrightarrow Y$ under $T$, the morphism $X / e_{e} T \longrightarrow Y / e \times{ }_{i^{*}(Y)} i^{*}(X)$ is a right fibration of simplicial sets.

In particular, for any $\infty$-operad $X$ under $T$, the map $X / e_{e} T \longrightarrow i^{*}(X)$ is a right fibration between $\infty$-categories. 
Proof. This follows from Proposition A.5 by a standard adjunction argument.

Theorem A.7. Let $S$ be a tree with at least two vertices, let $v$ be a unary top vertex in $S$, and let $p: X \longrightarrow Y$ be an inner Kan fibration between $\infty$-operads. Then any solid commutative square of the form

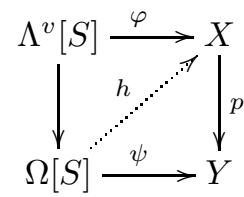

in which $\varphi(v)$ is weakly invertible in $X$ has a diagonal filling $h$.

Proof. The tree $S$ has to be of shape $S=1 \star_{e} T$ for a tree $T$ with a given leaf $e$. Under this identification, we have $\Lambda^{v}[S]=\Lambda^{0}\left[1 \star_{e} T\right]$. A lifting $h$ in the solid commutative square

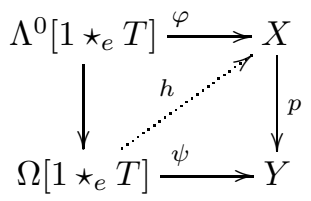

is thus equivalent to a lifting $k$ in the diagram

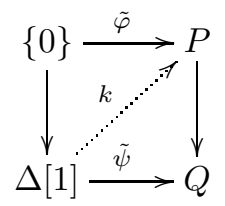

in which $P=X /{ }_{e} T$ and $Q=U \times_{W} V$, with

$$
U=\varliminf_{R}^{\lim _{R}} X /{ }_{e} R, \quad V=Y / e T, \quad W=\underbrace{\lim _{R}}_{R} Y /{ }_{e} R,
$$

where $R$ ranges over all the proper $e$-admissible faces of $T$. As in the proof of Theorem 4.2, it is now sufficient to prove the three following properties:

(i) the map $P \longrightarrow Q$ is a right fibration;

(ii) $Q$ is an $\infty$-category.

(iii) if $\varphi(x)$ is weakly invertible in $X$, then so is the 1 -cell $\tilde{\psi}$ in $Q$.

Properties (ii) and (iii) will follow from the two assertions below:

(iv) the map $V \longrightarrow W$ is a right fibration;

(v) the map $U \longrightarrow i^{*}(X)$ is a right fibration.

As (iv) is a particular case of (i), we are thus reduced to prove (i) and (v).

A.7.1. Proof of (i).

A lifting problem of shape

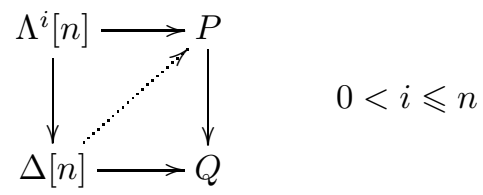


is equivalent to a lifting problem of shape

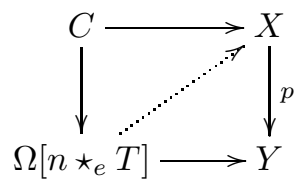

where $C$ is the union of $\Lambda^{i}[n] \star_{e} T$ with the union of the faces of $\Omega\left[n \star_{e} T\right]$ which are of the form $n \star_{e} S \longrightarrow n \star_{e} T$, where $S$ ranges over the $e$-admissible elementary faces of $T$. In other words, $C=\Lambda^{i}\left[n \star_{e} T\right]$ is an inner horn, so that the required lifting exists, because $p$ is assumed to be an inner Kan fibration.

A.7.2. Proof of (v).

A lifting problem of shape

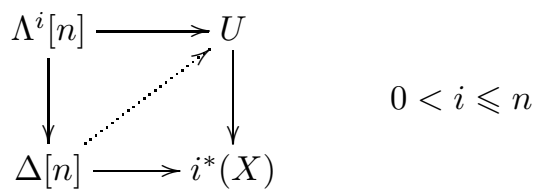

is equivalent to a lifting problem of shape

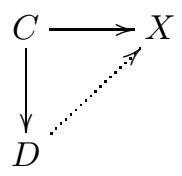

in which the inclusion $C \longrightarrow D$ can be described as follows:

$$
C=\Omega[n] \cup \bigcup_{R} \Lambda^{i}[n] \star_{e} R \subset D=\bigcup_{R} \Omega\left[n \star_{e} R\right] \subset \Omega\left[n \star_{e} T\right],
$$

where $R$ ranges over the $e$-admissible elementary faces of $T$. It is easily seen that the inclusion $C \longrightarrow D$ is an inner anodyne extension by Lemma A.4.

\section{B. Another SUbDivision OF CYLINDERS}

B.1. We will refer to the horn inclusions of shape $\Lambda^{x}[S] \longrightarrow \Omega[S]$, where $S$ is a tree with a unary top vertex $x$, as end extensions. A composition of pushouts of end extensions will be called an end anodyne map.

The goal of this section is to prove a dual version of Theorem 5.2, namely:

Theorem B.2. Let $T$ be a tree with at least one vertex, and consider the subobject

$$
B_{0}=\{0\} \otimes \Omega[T] \cup \Delta[1] \otimes \partial \Omega[T] \subset \Delta[1] \otimes \Omega[T] .
$$

There exists a filtration of $\Delta[1] \otimes \Omega[T]$ of the form

$$
B_{0} \subset B_{1} \subset \ldots \subset B_{N-1} \subset B_{N}=\Delta[1] \otimes \Omega[T]
$$

where, for each $i, 0 \leqslant i<N$, the map $B_{i} \longrightarrow B_{i+1}$ is either inner anodyne or end anodyne. 
Moreover, the end anodyne maps are all push outs of the form

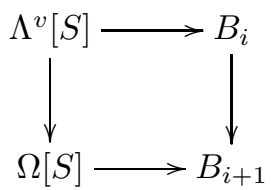

with the following properties:

(i) the tree $S$ has at least two vertices, and $v$ is a unary top vertex;

(ii) the map

$$
\Delta[1] \longrightarrow \Lambda^{v}[S] \longrightarrow B_{i} \subset \Delta[1] \otimes \Omega[T],
$$

corresponding to the vertex $v$ in $S$, coincides with an inclusion of shape

$$
\Delta[1] \otimes\{t\} \longrightarrow \Delta[1] \otimes \Omega[T]
$$

for some edge $t$ in $T$.

B.3. As in the proof of Theorem 5.2, we will follow the convention of MW09, and write

$$
\Omega[S] \otimes \Omega[T]=\bigcup_{i=1}^{m} \Omega\left[T_{i}\right]
$$

where the union ranges over the partially ordered set of percolation schemes, starting with a number of copies of $T$ grafted on top of $S$, and ending with the reverse grafting. For

$$
S=[1]=\oint
$$

the first tree is of shape

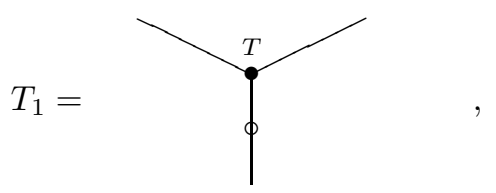

and the last one is

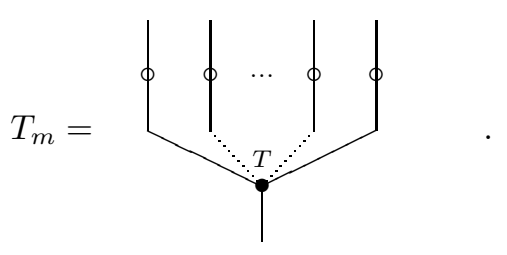

Let us fix a linear order on the percolation schemes for $\Delta[1] \otimes \Omega[T]$ which extends the natural partial order. Such a linear ordering induces a filtration on the tensor product $\Delta[1] \otimes \Omega[T]$,

$$
C_{0} \subset C_{1} \subset \ldots \subset C_{m-1} \subset C_{m}=\Delta[1] \otimes \Omega[T]
$$

by setting

(B.3.4) $C_{0}=B_{0}=\{0\} \otimes \Omega[T] \cup \Delta[1] \otimes \partial \Omega[T]$ and $C_{i}=B_{0} \cup \Omega\left[T_{1}\right] \cup \cdots \cup \Omega\left[T_{i}\right]$. the filtration of Theorem $\mathrm{B} .2$ will be a refinement of this one. 
Let us start by considering $T_{1}$. If the root edge of $T$ is called $r$, then $T_{1}$ looks like

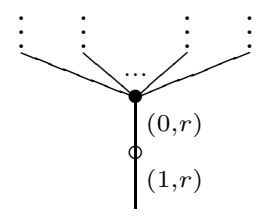

With the exception of the faces $\partial_{(0, r)}\left(T_{1}\right)$ (which contracts $(0, r)$ ) and $\partial_{(1, r)}\left(T_{1}\right)$ (which chops off $(1, r)$ as well as the white vertex), any face $F$ of $T_{1}$ misses a colour of $T$ (by this, we mean there is an edge $a$ in $T$ such that no edge in $F$ is named $(i, a)$ ). Hence, $\Omega[F] \subset \Delta[1] \otimes \partial \Omega[T]$ for these $F$. Moreover, $\partial_{(1, r)}\left(T_{1}\right)=\{0\} \otimes T_{1}$. So $\Omega\left[T_{1}\right] \cap B_{0}=\Lambda^{(0, r)}\left[T_{1}\right]$, and

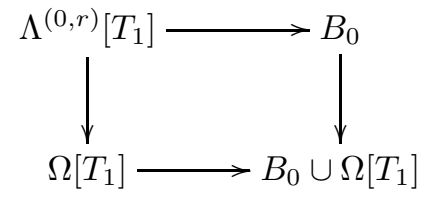

is a pushout. So, if we let $B_{1}=C_{1}$, then $B_{0} \longrightarrow B_{1}$ is obviously inner anodyne.

Suppose we have defined a filtration up to some $B_{l}$

$$
B_{0} \subset B_{1} \subset \ldots \subset B_{l} \quad l \geqslant 1,
$$

so that $B_{l}=C_{k}$ for some $k, 1 \leqslant k \leqslant m$. We will extend this filtration as $B_{l} \subset$ $B_{l+1} \subset \ldots \subset B_{l^{\prime}}$, so that $B_{l^{\prime}}=C_{k+1}$. The percolation scheme $T_{k+1}$ is obtained from an earlier one $T_{j}$ by pushing a white vertex in $T_{j}$ one step up through a black vertex $x$, as in

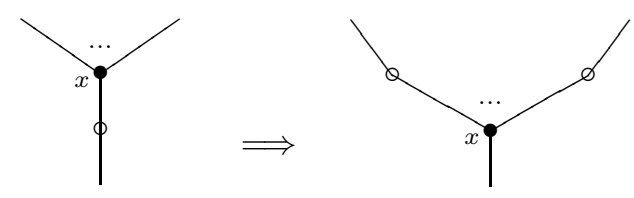

$$
\text { in } T_{j} \quad \text { in } T_{k+1}
$$

(we have denoted by $x$ the black vertex in both trees, although it would be more accurate to write $x$ for the relevant vertex of $T$, and write $0 \otimes x$ and $1 \otimes x$ for the corresponding vertices in $T_{j}$ and $T_{k+1}$ repectively). The Boardman-Vogt relation states that, as subobjects of $\Delta[1] \otimes \Omega[T]$, the face of $T_{k+1}$ obtained by contracting all input edges of $x$ coincides with the face of $T_{j}$ obtained by contracting the output edge of $x$ in $T_{j}$. In particular, notice that if $x$ has no input edges at all (i.e. if $x$ is a 'nullary operation' in $T$ ), then $T_{k+1}$ is a face of $T_{j}$, so $C_{k+1}=C_{k}$, and we let $B_{l^{\prime}}=B_{l}$, and there is nothing prove. Therefore, from now on, we will assume that the set of input edges of $x$, denoted $\operatorname{input}(x)$, is non-empty, and we proceed as follows.

Let $E$ be the set of all colours (edges) $e$ in $T$ for which

$$
\oint_{(1, e)}^{(0, e)}
$$


occurs in $T_{k+1}$. For $U \subset E$, let

$$
T_{k+1}^{(U)} \subset T_{k+1}
$$

be the face given by contracting all the edges $(1, e)$ for $e \in E$ but $e$ not in $U$. Notice that if $U \cap \operatorname{input}(x)=\varnothing$, then $\Omega\left[T_{k+1}^{(U)}\right] \subset B_{l}$ by the Boardman-Vogt relation just mentioned. Therefore, we will only consider $U$ with $U \cap \operatorname{input}(x) \neq \varnothing$. We will successively adjoin $\Omega\left[T_{k+1}^{(U)}\right]$ to $B_{l}$ for larger and larger such $U$, until we reach the case where $U=E$ and $T_{k+1}^{(U)}=T_{k+1}$.

If $U=\{e\}$ is a singleton (with $e$ an input edge of $x$ ), then the face $\partial_{(1, e)} \Omega\left[T_{k+1}^{(\{e\})}\right]$ is contained in $B_{l}$ as said, while the face $\partial_{(0, e)} \Omega\left[T_{k+1}^{(\{e\})}\right]$ is not (it cannot belong to an earlier $T_{i}$, and is obviously not contained in $B_{0}=C_{0}$ ). Any other face of $T_{k+1}^{(\{e\})}$ misses a colour of $T$ and hence is contained in $B_{0}$. Thus,

$$
\Omega\left[T_{k+1}^{(\{e\})}\right] \cap B_{l} \subset \Omega\left[T_{k+1}^{(\{e\})}\right]
$$

is either an inner horn (if $(0, e)$ is an inner edge of $\left.T_{k+1}\right)$ or an end extension (if $(1, e)$ is an input edge of $\left.T_{k+1}\right)$. In either case, we can adjoin $\Omega\left[T_{k+1}^{(\{e\})}\right]$ by forming the pushout below.

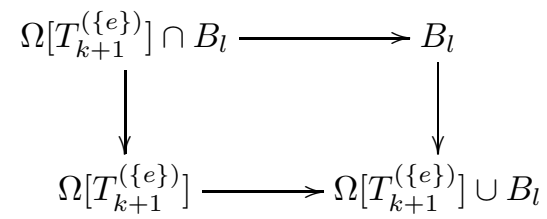

We successively adjoin $\Omega\left[T_{k+1}^{(\{e\})}\right]$ to $B_{l}$ in this way for all $e$ in $E$ which are input edges of $x$ in $T$ : if these are $e_{1}, \ldots, e_{p}$, let

$$
B_{l+r}=B_{l} \cup \Omega\left[T_{k+1}^{\left(\left\{e_{1}\right\}\right)}\right] \cup \cdots \cup \Omega\left[T_{k+1}^{\left(\left\{e_{r}\right\}\right)}\right] .
$$

Then, for each $r<p$, the map $B_{l+1} \longrightarrow B_{l+r+1}$ is inner anodyne or end anodyne.

In general, we proceed by induction on $U$. Choose $U \subset E$ with $U \cap \operatorname{input}(x) \neq \varnothing$, and assume we have adjoined $\Omega\left[T_{k+1}^{\left(U^{\prime}\right)}\right]$ already, for all $U^{\prime}$ of smaller cardinality than $U$. We will write $B_{l^{\prime \prime}}$ for the last object in the filtration constructed up to that point. Fix an order on the set of elements of $U$, and write it as

$$
U=\left\{\alpha_{1}, \ldots, \alpha_{s}\right\} .
$$

Consider $\Omega\left[T_{k+1}^{(U)}\right]$. The tree $T_{k+1}^{(U)}$ has edges $(0, c)$ or $(1, c)$ for $c$ not in $E$, and the corresponding face misses the colour $c$ alltogether, hence $\partial_{(i, c)} \Omega\left[T_{k+1}^{(U)}\right]$ is contained in $B_{0}$ for these $c$. Next, the tree $T_{k+1}^{(U)}$ has edges coloured $\left(1, \alpha_{i}\right)$ for $1 \leqslant i \leqslant s$, and contracting any of these gives a face

$$
\partial_{\left(1, \alpha_{i}\right)} \Omega\left[T_{k+1}^{(U)}\right]=\Omega\left[T_{k+1}^{\left(U-\left\{\alpha_{i}\right\}\right)}\right]
$$

which is contained in $B_{l^{\prime \prime}}$ by the inductive assumption on $U$. None of the faces given by contracting (if it is inner) or by chopping off (if it is outer) an edge $\left(0, \alpha_{i}\right)$ in $T_{k+1}^{(U)}$ can be contained in $B_{l^{\prime \prime}}$, however.

Let $A_{1}, \ldots, A_{t}$ be all the subsets of the set of these edges $\left\{\left(0, \alpha_{1}\right), \ldots,\left(0, \alpha_{s}\right)\right\}$ of $T_{k+1}^{(U)}$ which contain $\left(0, \alpha_{1}\right)$, and order them by some linear order extending the 
inclusion order. So there are $t=2^{s-1}$ such $A_{i}$, and we could fix the order to be

$$
\begin{aligned}
A_{1}= & \left\{\left(0, \alpha_{1}\right)\right\} \\
A_{2}= & \left\{\left(0, \alpha_{1}\right),\left(0, \alpha_{2}\right)\right\} \\
\vdots & \vdots \\
A_{s}= & \left\{\left(0, \alpha_{1}\right),\left(0, \alpha_{s}\right)\right\} \\
A_{s+1}= & \left\{\left(0, \alpha_{1}\right),\left(0, \alpha_{2}\right),\left(0, \alpha_{3}\right)\right\} \\
\vdots & \vdots \\
A_{t}= & \left\{\left(0, \alpha_{1}\right), \ldots,\left(0, \alpha_{s}\right)\right\} .
\end{aligned}
$$

For $q=1, \ldots, t$, let $T_{k+1}^{(U, q)}$ be the tree obtained from $T_{k+1}^{(U)}$ by contracting or chopping off all the edges $\left(0, \alpha_{i}\right)$ not in $A_{q}$. So

$$
T_{k+1}^{(U, 1)}=\partial_{\left(0, \alpha_{s}\right)} \partial_{\left(0, \alpha_{s-1}\right)} \ldots \partial_{\left(0, \alpha_{2}\right)} T_{k+1}^{(U)},
$$

and

$$
T_{k+1}^{(U, t)}=T_{k+1}^{(U)} .
$$

We will successively adjoin these $\Omega\left[T_{k+1}^{(U, q)}\right]$ to the filtration, to form the part

$$
B_{l^{\prime \prime}} \subset B_{l^{\prime \prime}+1} \subset \ldots \subset B_{l^{\prime \prime}+t}=B_{l^{\prime \prime}} \cup \Omega\left[T_{k+1}^{(U)}\right]
$$

of the filtration, as

$$
B_{l^{\prime \prime}+q}=B_{l^{\prime \prime}} \cup \Omega\left[T_{k+1}^{(U, 1)}\right] \cup \ldots \Omega\left[T_{k+1}^{(U, q)}\right] .
$$

We start with $T_{k+1}^{(U, 1)}$. The only face of $\Omega\left[T_{k+1}^{(U, 1)}\right]$ not contained in $B_{l^{\prime \prime}}$ is the one given by the edge $\left(0, \alpha_{1}\right)$. Thus

$$
\Omega\left[T_{k+1}^{(U, 1)}\right] \cap B_{l^{\prime \prime}} \subset \Omega\left[T_{k+1}^{(U, 1)}\right]
$$

is either an inner horn (if $\left(0, \alpha_{1}\right)$ is an inner edge) or an end extension (if $\left(0, \alpha_{1}\right)$ is an input edge of $T_{k+1}^{(U)}$, i.e. $\alpha_{1}$ is an input edge of $T$ ). So the pushout $B_{l^{\prime \prime}} \longrightarrow B_{l^{\prime \prime}+1}$ is either inner anodyne or end anodyne.

Suppose we have adjoined $\Omega\left[T_{k+1}^{\left(U, q^{\prime}\right)}\right]$ for all $1 \leqslant q^{\prime}<q$, so have arrived at the stage $B_{l^{\prime \prime}+q-1}$ of the filtration. Consider now $A_{q}$ and the corresponding dendroidal set $\Omega\left[T_{k+1}^{(U, q)}\right]$. As before, its faces given by edges coloured by $(i, c)$ for $i=0,1$ with $c$ not in $E$ are contained in $B_{0}$, and its faces given by edges coloured $(1, e)$ with $e \in U$ are contained in $\Omega\left[T_{k+1}^{\left(U^{\prime}\right)}\right]$ for a smaller $U^{\prime}=U-\{e\}$, hence are contained in $B_{l^{\prime \prime}}$. Let us consider the remaining faces given by the edges $\left(0, \alpha_{1}\right), \ldots,\left(0, \alpha_{r}\right)$ in $A_{q}$. If $i \neq 1$, the face of $\Omega\left[T_{k+1}^{(U, q)}\right]$ given by $\left(0, \alpha_{i}\right) \in A_{q}$ is contained in $\Omega\left[T_{k+1}^{\left(U, q^{\prime}\right)}\right]$ for some $q^{\prime}<q$ with $A_{q^{\prime}}=A_{q}-\left\{\left(0, \alpha_{i}\right)\right\}$. So the only face that is missing is the one given by $\left(0, \alpha_{1}\right)$, i.e.

$$
\Omega\left[T_{k+1}^{(U, q)}\right] \cap B_{l^{\prime \prime}+q-1}=\Lambda^{\left(0, \alpha_{1}\right)}\left[T_{k+1}^{(U, q)}\right] .
$$

Therefore, the induced pushout $B_{l^{\prime \prime}+q-1} \longrightarrow B_{l^{\prime \prime}+q}$ is either inner anodyne or end anodyne (depending on whether $\alpha_{1}$ is an external edge of $T$ or not). At the end, when $q=t$, we have adjoined all of $\Omega\left[T_{k+1}^{(U)}\right]$. 
This completes the contruction of the segment of the filtration for the subset $U \subset E$. As said, we continue this construction until we reach the stage $U=E$, when $T_{k+1}^{(U)}=T_{k+1}$, which completes the construction of the segment of the filtration from $B_{l}$ until $B_{l^{\prime}}$, interpolating between $C_{k}$ and $C_{k+1}$. This completes the description of the filtration. From it, the last part of the theorem is clear.

\section{ERRATUM}

Proposition 1.9 is wrong as stated, and should be modified as we will explain below. This modification does not affect any of the main results of this paper and its two sequels CM13a, CM13b: the existence of the model structure on dendroidal sets of the present paper, the equivalent model structures for dendroidal complete Segal spaces and for Segal operads in [CM13a], and the Quillen equivalence to the model category of simplicial operads in CM13b. However, the error does affect all the statements concerning the monoidality of the model structures.

Recall the inclusion $i: \Delta \rightarrow \Omega$, and the induced left Quillen functor $i_{!}: s \mathcal{S} e t \rightarrow$ $d S e t$. Let us call a tree (an object of $\Omega$ ) linear if it lies in the image of $i$, and a dendroidal set simplicial if it lies in the image of $i_{\text {! }}$. Also, let us call a tree open if it has no vertices of valence zero (i.e. no vertices without input edges). If $S \rightarrow T$ is a morphism in $\Omega$ and $T$ is open, then $S$ is necessarily open as well. Therefore, the open trees define a subobject $U$ of the terminal object in $d S e t$. Let us call a dendroidal set open if the unique map to the terminal object factors through $U$. These open dendroidal sets form a full subcategory $d S$ Set/U of $d S$ Set. More generally, we call a normal monomorphism $X \rightarrow Y$ linear if it is obtained by attaching linear trees (i.e. $X \rightarrow Y$ lies in the saturation of $\partial \Omega[T] \rightarrow \Omega[T]$ for $T$ linear) and open if it is obtained by attaching open trees.

The modified version of Proposition 1.9 should be:

Proposition 1.9. Let $A \rightarrow B$ and $X \rightarrow Y$ be normal monomorphisms. If one of them is linear or both are open, then the induced map

$$
A \otimes Y \cup_{A \otimes X} B \otimes X \rightarrow B \otimes Y
$$

is again a normal monomorphism (and is open as well in the second case).

By the usual induction, this follows from the following lemma (whose proof is an elementary but tedious combinatorial argument, and we refer the reader to CMn for the details), which should be added at the very end of the first section:

Lemma 1.11. Let $S$ and $T$ be trees. If one of them is linear or both are open, then the pushout-product map

$$
\partial \Omega[S] \otimes \Omega[T] \cup_{\partial \Omega[S] \otimes \partial \Omega[T]} \Omega[S] \otimes \partial \Omega[T] \rightarrow \Omega[S] \otimes \Omega[T]
$$

is a normal monomorphism (and is open as well in the second case).

The main result of [MW09] (namely Proposition 9.2) is wrong as stated, but its proof says the following: given two trees $S$ and $T$, as well as an inner edge $e$ of $S$, if $W$ denotes the the image of the map

$$
\Lambda^{e}[S] \otimes \Omega[T] \cup_{\Lambda^{e}[S] \otimes \partial \Omega[T]} \Omega[S] \otimes \partial \Omega[T] \rightarrow \Omega[S] \otimes \Omega[T],
$$

then the inclusion

$$
W \subset \Omega[S] \otimes \Omega[T]
$$


is inner anodyne. This means that Proposition 3.1 should be replaced by the following statement.

Proposition 3.1. Let $A \rightarrow B$ and $X \rightarrow Y$ be normal monomorphisms. If one of them is linear or both are open, and if one of them is inner anodyne, then the induced map

$$
A \otimes Y \cup_{A \otimes X} B \otimes X \rightarrow B \otimes Y
$$

is again an inner anodyne extension.

Proposition 3.3 should be modified accordingly (replacing, in Proposition 3.1 above, the expression "inner anodyne" by " $J$-anodyne").

Let us call a model category $\mathcal{M}$ Joyal simplicial if it satisfies the axioms for a simplicial model category, but with respect to the Joyal model structure on simplical sets instead of the classical Kan-Quillen structure. The model structure on dSet established in this paper is not monoidal. Instead, Proposition 3.17 should be replaced by the following result, which follows immediately from the propositions above and from the arguments explained in the original 'proof' of Prop. 3.17:

Proposition 3.17. The Boardman-Vogt tensor product turns the model structure on dSet into a Joyal simplicial model structure, and induces a symmetric monoidal model structure on the category dSet/U of open dendroidal sets.

In the proof of the existence of the model structure, it is only the "linear half" of Propositions 1.9 and 3.1 which are used, and this proof is unaffected. Indeed, Propositions 3.1 and 3.3 are used only in the case where one of the maps is in the image of $i_{!}$.

We close this erratum with a list of places where the reference to (consequences of) monoidality should be reformulated in accordance with the previous two propositions: In the present paper, these are point 1 in the introduction, Proposition 2.6(c), Corollary 2.8(b), Corollary 2.9(b); In the proof of Lemma 6.15, the first sentence should begin as 'We know that the image by the functor $i^{*}$ of the map...'; Also the statement between brackets at the very end of Proposition 6.20 should be skipped (and the proof of Prop. 6.20 should refer to Boardman and Vogt's explicit description of the category associated to a quasi-category, instead of its dendroidal analogue). In CM13a, CM13b, no use of the alleged monoidality of the model structure is made, and the necessary changes all concern inessential references to the monoidality: in paper CM13a, the places where we recall the monoidality from the present paper, are in the abstract and the introduction, in Theorem 1.1, Remarks 6.14 and 8.16, and finally in the proof of Propposition 6.11, where it is Proposition 3.1 above which should be used rather than the monoidality; and in paper CM13b], the mentioning of monoidality is in the introduction as well as in Proposition 2.8 and Theorem 5.7.

We repeat that this error only affects the monoidality of the model structures, and none of the main results about the existence of the model structure on dSet and the Quillen equivalent model categories presented in CM13a, CM13b.

\section{REFERENCES}

[Ber07] J. Bergner, Three models for the homotopy theory of homotopy theories, Topology 46 (2007), 397-436. 
[BV73] J. M. Boardman and R. M. Vogt, Homotopy invariant algebraic structures on topological spaces, Lecture Notes in Math., vol. 347, Springer-Verlag, 1973.

[Cis06] D.-C. Cisinski, Les préfaisceaux comme modèles des types d'homotopie, Astérisque, vol. 308, Soc. Math. France, 2006.

[CM13a] _ Dendroidal Segal spaces and $\infty$-operads, J. Topol. 6 (2013), no. 3, 675-704.

[CM13b] — Dendroidal sets and simplicial operads, J. Topol. 6 (2013), no. 3, 705-756.

[CMn] - Note on the tensor product of dendroidal sets.

[Cra95] S. E. Crans, Quillen closed model structures for sheaves, J. Pure Appl. Algebra 101 (1995), 35-57.

[GZ67] P. Gabriel and M. Zisman, Calculus of fractions and homotopy theory, Ergebnisse der Mathematik, vol. 35, Springer-Verlag, 1967.

[Hov99] M. Hovey, Model categories, Math. surveys and monographs, vol. 63, Amer. Math. Soc., 1999.

[Joy02] A. Joyal, Quasi-categories and Kan complexes, J. Pure Appl. Algebra 175 (2002), no. 1$3,207-222$.

[JT07] A. Joyal and M. Tierney, Quasi-categories vs Segal spaces, Categories in Algebra, Geometry and Physics, Contemp. Math., vol. 431, Amer. Math. Soc., 2007, pp. 277-326.

[Lur06] J. Lurie, Higher topos theory, arXiv:math/0608040, 2006.

[MW07] I. Moerdijk and I. Weiss, Dendroidal sets, Algebraic \& Geometric Topology 7 (2007), $1441-1470$

[MW09] - On inner Kan complexes in the category of dendroidal sets, Adv. Math. 221 (2009), no. 2, 343-389.

[Wei07] I. Weiss, Dendroidal sets, PhD thesis, Universiteit Utrecht, 2007.

LAGA, CNRS (UMr 7539), Université Paris 13, Avenue Jean-Baptiste Clément, 93430 VilLETANEUSE, France

E-mail address: cisinski@math.univ-paris13.fr

URL: http://www.math.univ-paris13.fr/ cisinski/

Mathematisch Institudt, Universiteit Utrecht, PO.Box 80.010, 3508 TA Utrecht, The Netherlands

E-mail address: moerdijk@math.uu.nl

URL: http://www.math.uu.nl/people/moerdijk/ 